bioRxiv preprint doi: https://doi org/10.1101/2020.12 07 415257; this version posted December 7, 2020. The copyright holder for this preprint (which was not certified by peer review) is the author/funder, who has granted bioRxiv a license to display the preprint in perpetuity. It is made available under aCC-BY 4.0 International license.

\title{
Identifying a GSK3-TRARG1 signaling axis reveals BCL9L as a novel regulator of GLUT4 trafficking
}

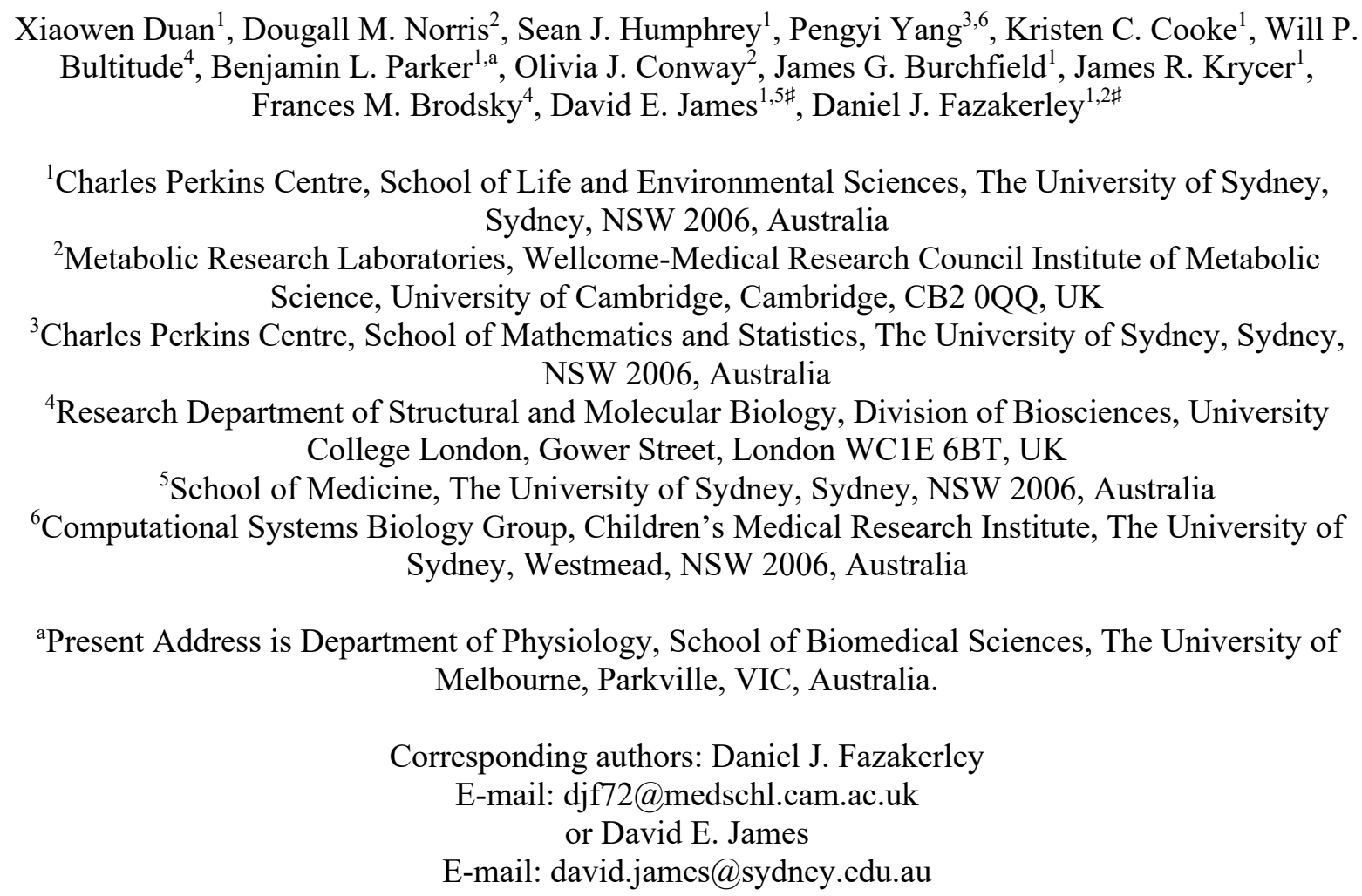

Running title: TRARG1 is a novel substrate of GSK3

Keywords: glycogen synthase kinase 3 (GSK3), glucose transporter type 4 (GLUT4), trafficking regulator of GLUT4-1 (TRARG1), protein phosphorylation, phosphoproteomics, proteomics, insulin signaling, protein-protein interaction, BCL9L 
bioRxiv preprint doi: https://doi.org/10.1101/2020.12.07.415257; this version posted December 7, 2020. The copyright holder for this preprint (which was not certified by peer review) is the author/funder, who has granted bioRxiv a license to display the preprint in perpetuity. It is made available under aCC-BY 4.0 International license.

\begin{abstract}
Trafficking regulator of GLUT4-1, TRARG1, positively regulates insulin-stimulated GLUT4 trafficking and insulin sensitivity. However, the mechanism(s) by which this occurs remain(s) unclear. Using biochemical and mass spectrometry analyses we found that TRARG1 is dephosphorylated in response to insulin in a PI3K/Akt-dependent manner and is a novel substrate for GSK3. Priming phosphorylation of mouse TRARG1 at serine 84 allows for GSK3directed phosphorylation at serines 72,76 and 80 . A similar pattern of phosphorylation was observed in human TRARG1, suggesting that our findings are translatable to human TRARG1. Pharmacological inhibition of GSK3 increased cell surface GLUT4 in cells stimulated with a submaximal insulin dose, and this was impaired following Trargl knockdown, suggesting that TRARG1 acts as a GSK3-mediated regulator in GLUT4 trafficking. TRARG1 dephosphorylation in response to insulin or GSK3 inhibition regulated its protein-protein interactions, decreasing the interaction between TRARG1 and BCL9L, JAGN1, PYGO2 and HSD17B12. Knock-down of Bcl9l promoted GLUT4 translocation to the plasma membrane. These data place TRARG1 within the insulin signaling network and provide insights into how TRARG1 regulates GLUT4 trafficking in adipocytes.
\end{abstract}

\section{Introduction}

Insulin maintains whole-body glucose homeostasis by regulating glucose transport into muscle and adipose tissue and by inhibiting glucose output from liver. Defects in these processes cause insulin resistance, which is a precursor to metabolic disorders including Type 2 diabetes. Insulin signaling augments glucose transport into adipose tissue and muscle by promoting the translocation of the glucose transporter GLUT4 from a specialized intracellular storage compartment (GLUT4 storage vesicles; GSVs) to the plasma membrane (PM) [1]. Previous proteome studies of intracellular membranes highly enriched in GLUT4 have revealed major GSV resident proteins including TBC1D4 (AS160), Sortilin, IRAP, LRP1, VAMP2 [2-4] and Trafficking regulator of GLUT4-1 (TRARG1). TRARG1 positively regulated insulin-stimulated glucose transport and GLUT4 trafficking, and was required for the insulin-sensitizing action of the PPAR $\gamma$ agonist rosiglitazone $[5,6]$. However, the mechanisms by which insulin signals to TRARG1 or how TRARG1 promotes insulin sensitivity remains unknown.

The most characterized signaling pathway relevant to GLUT4 trafficking is the PI3K/Akt signaling axis, which is activated by a series of upstream signaling events triggered by the binding of insulin to its receptor at the cell surface. Akt is necessary and sufficient for insulinstimulated GLUT4 trafficking [7], and is thought to act predominantly via phosphorylation of the Rab GTPase-activating protein (GAP), TBC1D4. Phosphorylation of TBC1D4 inhibits its GAP activity, and permits its target Rab GTPases to mediate the translocation of GSVs to the PM [8]. However, loss of TBC1D4 or its cognate Rab proteins did not completely mimic or inhibit insulin-stimulated GLUT4 translocation [9-11], suggesting that there may be additional sites of insulin action within the GLUT4 trafficking pathway [12].

In addition to phosphorylating substrates such as TBC1D4, Akt also modulates cellular metabolism through increased or diminished activity of other kinases. For example, Akt directly phosphorylates glycogen synthase kinase 3 (GSK3) at the regulatory Ser 21 or 9 residue $(\alpha$ and $\beta$ isoform, respectively), which inhibits its kinase activity [13] and leads to dephosphorylation and activation of glycogen synthase (GS) and thereby glycogen synthesis. GSK3 is an unique kinase in that 1) it is constitutively active and inhibited in response to extracellular stimulation and 2) its substrates usually need priming by another kinase [14]. Given the role of GSK3 in glycogen synthesis, it has generally been thought that GSK3 mainly participates in glucose metabolism via regulation of glycogen synthesis [15-19]. However, although there are conflicting reports [20-22], evidence from studies using small molecules to acutely inactivate GSK3 shows that GSK3 may also regulate glucose transport and GLUT4 translocation $[21,22]$.

In the present study, we identified a range of posttranslational modifications (PTMs) on TRARG1 and integrated TRARG1 into the insulin signaling pathway by showing that insulin promotes TRARG1 dephosphorylation through the PI3K/Akt axis. Specifically, we report that 
bioRxiv preprint doi: https://doi.org/10.1101/2020.12.07.415257; this version posted December 7, 2020. The copyright holder for this preprint (which was not certified by peer review) is the author/funder, who has granted bioRxiv a license to display the preprint in perpetuity. It is made available under aCC-BY 4.0 International license.

TRARG1 is a novel direct downstream substrate of the protein kinase GSK3, phosphorylating murine TRARG1 at three sites within a highly phosphorylated patch between residues 70 and 90 in the cytosolic portion of TRARG1. Further, potentiation of submaximal insulin-stimulated GLUT4 trafficking induced by GSK3 inhibition was impaired by Trargl knockdown. GSK3mediated TRARG1 phosphorylation did not alter TRARG1 subcellular localization but regulated TRARG1 protein-protein interactions (PPIs). TRARG1 interactors, for example BCL9L, a transcriptional co-activator of $\beta$-catenin, played a negative role in GLUT4 translocation to the PM. These findings have revealed new information on how TRARG1 is regulated by insulin and provided insights into how it may regulate GLUT4 trafficking in adipocytes.

\section{Results}

\section{TRARG1 phosphorylation altered its migration in SDS-PAGE}

We previously reported that a proportion of TRARG1 exhibited reduced electrophoretic mobility following separation by SDS-PAGE. These apparent higher molecular weight species of TRARG1 were enriched in the PM but not low or high density microsomal (LDM, HDM) fractions (Fig. S1A), and only present in epididymal and subcutaneous white adipose depots, but not in brown adipose tissue (Fig. S1B) [5]. Given the role of TRARG1 in GLUT4 trafficking, we sought to identify the cause of altered electrophoretic mobility of TRARG1 with the aim of providing insight into how TRARG1 regulates GLUT4. We hypothesized that these apparent higher molecular weight TRARG1 bands are due to PTM of TRARG1 as: 1) they were all reduced in intensity upon knock-down of TRARG1 using different sets of siRNAs [5], and 2) they are not splice variants as the cDNA of TRARG1, which does not contain any introns, also generates multiple bands when transfected into HEK-293E cells (Fig. 1A).

We first used mass spectrometry (MS) analysis to analyze PTMs on immunoprecipitated HAtagged TRARG1 (HA-TRARG1) expressed in HEK-293E cells. This revealed multiple PTM types on mouse TRARG1 including phosphorylation, methylation, oxidation, ubiquitination (or other ubiquitin-like modifications as indicated by peptides containing di-gly modifications) and acetylation (Fig. 1B, Supplemental table S1). This analysis revealed TRARG1 to be extensively post-translationally modified, with a particularly high number of phosphorylated sites within a short cytosolic region between residues 70 and 90 . To test if these specific PTMs affected TRARG1 mobility in SDS-PAGE, we generated a series of HATRARG1 mutants, including Ser/Thr to Ala/Glu mutants (7A and 7E mutants where S74, S76, S79, $\mathrm{S} 84, \mathrm{~S} 85, \mathrm{~T} 88, \mathrm{~S} 90$ were mutated to A or E; or $12 \mathrm{~A}$ and $11 \mathrm{E}$ mutants where all $\mathrm{S} / \mathrm{T}$ sites between S70 and S90 were mutated to A or E, with the 12A mutant containing an extra Y91 mutated to A) where $\mathrm{A}$ prevents and $\mathrm{E}$ mimics phosphorylation [23], a Lys-null mutant $(\mathrm{K} \rightarrow \mathrm{R})$ to prevent ubiquitination or acetylation of Lys and a Cys-null mutant $(\mathrm{C} \rightarrow \mathrm{S})$ to prevent palmitoylation of Cys. Of these mutants, only the Ser/Thr to Ala mutant expressed in HEK-293E cells or 3T3-L1 adipocytes exhibited a similar electrophoretic mobility as the lower band of HATRARG1, while Ser/Thr to Glu mutant migrated to a similar position as the higher bands of HATRARG1 (Fig. 1C and S1C). These data suggest that the apparent higher molecular weight TRARG1 bands are dependent on PTMs on Ser/Thr, but not Lys or Cys.

Consistent with these data, treatment of lysates from 3T3-L1 adipocytes (Fig. 1D), HEK-293E cells (Fig. S1D) transfected with HA-TRARG1 or adipose tissue (Fig. S1B) with Lambda protein phosphatase (LPP) in vitro completely removed apparent higher molecular weight TRARG1 bands. Further, acute treatment of 3T3-L1 adipocytes with the phosphatase inhibitors Calyculin A or Okadaic Acid decreased TRARG1 migration in SDS-PAGE (Fig. 1E-F). Therefore, TRARG1 is extensively post-translationally modified, with phosphorylation reducing TRARG1 mobility by SDS-PAGE. Additionally, the ratio of apparent higher molecular weight TRARG1 signal to total TRARG1 signal by immunoblotting can serve as a proxy metric for TRARG1 phosphorylation (Fig. 1F).

\section{TRARG1 is dephosphorylated with insulin in a PI3K/Akt-dependent manner}

Given the role of TRARG1 in adipocyte insulin responses [5, 6], we next tested whether TRARG1 phosphorylation was regulated by insulin signaling. Using the ratio of apparent higher molecular weight TRARG1 versus total 
bioRxiv preprint doi: https://doi.org/10.1101/2020.12.07.415257; this version posted December 7, 2020. The copyright holder for this preprint (which was not certified by peer review) is the author/funder, who has granted bioRxiv a license to display the preprint in perpetuity. It is made available under aCC-BY 4.0 International license.

TRARG1 as a proxy for TRARG1 phosphorylation, we detected a decrease in TRARG1 phosphorylation in 3T3-L1 adipocytes stimulated with insulin (Fig.2 A and B). This decrease was blocked by PI3K (wortmannin) or Akt inhibition (MK-2206), but not by mTORC1 (rapamycin) or ERK1/2 inhibition (GDC-0994) (Fig. 2A and B). As evidence for the efficacy of kinase inhibition, phosphorylation of Akt S473 and TBC1D4 T642 was impaired by wortmannin and MK-2206; 4EBP1 S65 by wortmannin, MK2206 and rapamycin; and p90RSK T359/S363 by GDC-0994 (Fig. 2A). These data indicate that TRARG1 is dephosphorylated in response to insulin in a PI3K/Akt-dependent manner. To complement this, we mined data from a SILACbased, global phosphoproteomic analysis of insulin-stimulated 3T3-L1 adipocytes previously published by our laboratory [24]. These data revealed five phosphosites on TRARG1 (Ser45, Ser76, Ser79, Ser80 and Ser90) downregulated $\leq$ 0.67 -fold with insulin treatment compared to basal conditions (Fig. 2C). The most insulinsensitive sites were Ser76, Ser79 and Ser80 (Fig. 2C). Akt inhibition using MK-2206 before insulin stimulation increased the phosphorylation at Ser76 and Ser80 by $\geq 1.5$-fold compared to insulin stimulation alone (Fig. 2C). These data suggest that phosphosites on TRARG1, probably S76 and S80, are dephosphorylated in response to insulin in a PI3K/Akt-dependent manner.

\section{TRARG1 is a novel substrate of GSK3}

To complete the chain of events by which insulin decreases TRARG1 phosphorylation, we next aimed to identify the kinase responsible for TRARG1 phosphorylation. Here, we considered PKA or GSK3; kinases deactivated by insulin. To test for the involvement of PKA in TRARG1 phosphorylation, we treated 3T3-L1 adipocytes with insulin and/or isoproterenol, a $\beta$-adrenergic receptor agonist that potently activates PKA. Insulin-stimulation decreased phosphorylation of both TRARG1 and HSL, a PKA substrate (Fig. 2D). Isoproterenol rescued HSL phosphorylation, but not TRARG1 phosphorylation, suggesting that TRARG1 is not a PKA substrate. In contrast, the GSK3 inhibitors, CHIR99021 and LY2090314, both lowered phosphorylation of glycogen synthase at S641, a GSK3 substrate, and decreased TRARG1 phosphorylation (Fig. 2E). The same effect was observed on endogenous TRARG1 in epididymal (EWAT) and subcutaneous (SWAT) fat explants, and cultured human SGBS adipocytes, treated with insulin or GSK3 inhibitors (Fig. 2F-G and S2A-C), and also on ectopically expressed TRARG1 in HEK-293E cells, HeLa cells and L6 myotubes treated with GSK3 inhibitors (Fig. S2D-F).

To complement this pharmacological approach, and to test for a GSK3 isoform-specific effect on TRARG1 phosphorylation, we knocked down GSK3 $\alpha$ and/or GSK3 $\beta$, in 3T3-L1 adipocytes and assessed the phosphorylation status of TRARG1 (Fig. 2H, Fig. S2G). Phosphorylation of glycogen synthase was only significantly decreased with the knock down of both Gsk3 isoforms (Fig. 2H and Fig. S2G). Phosphorylation of TRARG1 was significantly decreased under conditions where Gsk $3 \beta$ was depleted (Fig. 2H-I), but knockdown of $G s k 3 \alpha$ alone did not affect TRARG1 phosphorylation (Fig. 2H-I). These data suggest that TRARG1 is predominantly phosphorylated by GSK $3 \beta$ in 3T3-L1 adipocytes.

We next utilized an in vitro assay to test if GSK3 phosphorylated TRARG1 directly. Endogenous TRARG1 was immunoprecipitated from serumstarved 3T3-L1 adipocytes treated with LY2090314 to ensure that the majority of TRARG1 was dephosphorylated while the priming site remained phosphorylated and incubated with recombinant GSK $3 \alpha$ or GSK $3 \beta$. Incubation with either GSK3 isoform decreased TRARG1 electrophoretic mobility (Fig. 2J), suggesting that TRARG1 was phosphorylated by both GSK $3 \alpha$ and GSK $3 \beta$ in vitro. Together, these data suggest that TRARG1 phosphorylation is regulated by insulin through the PI3K-Akt-GSK3 axis.

Given the role of TRARG1 in insulin-stimulated GLUT4 trafficking, and previous reports that inhibition of GSK3 potentiates insulin-stimulated GLUT4 trafficking [21, 22], we tested whether TRARG1 played a role in GSK3 inhibitionpromoted GLUT4 trafficking. Inhibition of GSK3 with either CHIR99021 or LY2090314 increased cell surface GLUT4 under insulin-stimulated conditions, compared to DMSO control cells (Fig. 2K). Trargl knockdown significantly decreased submaximal and maximal insulin-stimulated PM GLUT4 compared to cells treated with nontargeting (NT) siRNA control (Fig. 2K), as previously reported $[5,6]$. To determine whether Trarg1 depletion blunts the effect of GSK3 inhibitors on GLUT4 trafficking, we calculated the difference in PM GLUT4 between 
bioRxiv preprint doi: https://doi.org/10.1101/2020.12.07.415257; this version posted December 7, 2020. The copyright holder for this preprint (which was not certified by peer review) is the author/funder, who has granted bioRxiv a license to display the preprint in perpetuity. It is made available under aCC-BY 4.0 International license.

CHIR99021- or LY2090314-treated cells and cells treated with DMSO under the same knockdown and insulin treatment conditions (Fig. 2L). The increase in PM GLUT4 in submaximal $(0.5 \mathrm{nM})$ insulin-stimulated adipocytes in response to GSK3 inhibitors was blunted by Trarg1 knockdown (Fig. 2L). However, GSK3i increased cell surface GLUT4 to a similar extent in both control and Trarg1 KD adipocytes in response to maximal insulin stimulation (Fig. 2L). These data suggest that GSK3 activity regulates GLUT4 trafficking, and that GSK3 inhibition may increase GLUT4 trafficking to the PM at submaximal insulin doses in part via TRARG1.

\section{Identification of GSK3 target sites on TRARG1}

To identify the GSK3 sites on TRARG1, we first performed a phosphoproteomic study with GSK3 inhibitor LY2090314. As expected, phosphorylation of the known GSK3 sites on glycogen synthase (S649, S645 and S641) were significantly decreased with GSK3 inhibition (Fig. 3A). On TRARG1, S72 was the only site significantly dephosphorylated with GSK3 inhibition among the sites detected (Fig. 3A). In general, GSK3 has a substrate motif where it can only phosphorylate an amino acid if there exists a pre-phosphorylated (primed by a different kinase) $\mathrm{S} / \mathrm{T}$ four residues $\mathrm{C}$-terminal to the target site (Fig. 3B) [14]. Substrates of GSK3 often contain three or four adjacent $\mathrm{S} / \mathrm{T}-\mathrm{X}-\mathrm{X}-\mathrm{X}-\mathrm{pS} / \mathrm{T}$ motifs, allowing GSK3 to phosphorylate every fourth residue in a string of sequential sites as it creates its own primed site in a stepwise fashion [14]. T88, S84, S80, S76 and S72 formed such a putative string of sequential sites on TRARG1. Although phosphorylation of S80 and S76 were not detected in this study (Fig. 3A), they were both decreased by insulin and rescued by an Akt inhibitor (Fig. 2C), which matches the attributes of GSK3 target sites. Of note, S72 was not regulated by insulin in the phosphoproteomic analysis described in Fig. 2C [24] although it was clearly down-regulated in response to GSK3 inhibition (Fig. 3A). Since phosphorylation of neither S84 nor T88 was significantly regulated by the GSK3 inhibitor, we hypothesized that S84 or S88 is the priming site (pre-phosphorylated by a different kinase). To test this, we expressed mouse TRARG1 phosphomutants with $\mathrm{S} / \mathrm{T}$ at positions $79 / 80,84,85$, or $88 / 89 / 90$ mutated to Ala in HEK-293E cells. Only TRARG1 with S84 mutated to Ala lost the apparent higher molecular weight bands (Fig. 3C), suggesting that S84 is the priming site on mouse TRARG1 allowing for its subsequent phosphorylation by GSK3 at multiple sites (S80, S76 and S72), which altered TRARG1 migration in SDS-PAGE. In support of this, mouse TRARG1 with S72/76/80 mutated to Ala completely lost the apparent higher molecular weight bands, similar to the S84A mutant (Fig. 3D).

Since functionally important phosphosites are more likely conserved across species [25], we aligned TRARG1 sequences from multiple species in which TRARG1 homologues were identified. The phosphosite-rich region between residues 70 and 90 in the mouse sequence is highly conserved across species (Fig. 3E; pink and red residues), indicating a likely important function for this region. Of the insulin/GSK3 regulated sites identified from phosphoproteomic analyses (Fig. 3E, red residues), S76 (human S79) was conserved across all species tested, although S80 appeared to be more specific to placental mammals. Analysis of TRARG1 polymorphisms across placental mammals (Fig. 3F, Supplemental table S2) revealed that mouse S76, S79, S80, S84, S85, T88 and S90 (human S79, S82, S83, S87, S88, T91 and S93) were conserved within placental mammals, while mouse S72 was only present in rodents (Supplemental table S2). Indeed, the Ala mutant of S87 on human TRARG1, which is equivalent to the S84 priming site on mouse TRARG1, abolished its apparent higher molecular weight bands (Fig. 3G), suggesting that this conserved site acts as the equivalent priming site for human TRARG1 phosphorylation by GSK3.

Taken together, our data show that mouse TRARG1 is primed at S84 for subsequent phosphorylation by GSK3 at S80, S76 and S72. Further, these sites are highly conserved across species including human with the exception of S72, which is only present in some rodents.

\section{TRARG1 phosphorylation regulates its interactome but not subcellular distribution}

Since we initially observed the apparent higher molecular weight bands of TRARG1 were highly enriched within the PM subcellular fraction (Fig. S1A), and TRARG1 undergoes insulinstimulated translocation to the PM [5], we determined whether TRARG1 phosphorylation status altered its localization. To this end, we 
bioRxiv preprint doi: https://doi.org/10.1101/2020.12.07.415257; this version posted December 7, 2020. The copyright holder for this preprint (which was not certified by peer review) is the author/funder, who has granted bioRxiv a license to display the preprint in perpetuity. It is made available under aCC-BY 4.0 International license.

performed immunofluorescence microscopy with HA-TRARG1 or 7A/7E phosphomutants expressed in 3T3-L1 adipocytes. These mutants targeted the majority of the most highly conserved residues within the 70-90 phosphositerich region of mouse TRARG1 to mitigate redundancy between phosphosites. Although the S72 and S80 sites were not mutated in this construct, the $7 \mathrm{~A}$ or $7 \mathrm{E}$ mutant mimicked the lower (dephosphorylated form) or higher (phosphorylated form) band of TRARG1, respectively (Fig. 1C), likely because of the requirement for phosphorylation of S84 for GSK3-mediated phosphorylation of S72, and S80 (Fig. 3C-D). HA-TRARG1 and GLUT4 colocalized at the PM and in the peri-nuclear region (Fig. 4A), consistent with previous reports [5]. The subcellular distribution of HA-TRARG1-7A and HA-TRARG1-7E were indistinguishable from HA-TRARG1 being localized to the PM and peri-nuclear regions (Fig. 4A). These data suggest that the phosphorylation status of TRARG1 does not alter its localization.

As part of our previous characterization of TRARG1 topology [26], we revealed that the Nterminal cytosolic domain of TRARG1 (1-100; del_101-173) does not exhibit reduced migration by SDS-PAGE, suggesting that this truncation mutant is not phosphorylated (Fig. S3A-B). In contrast, membrane-associated truncations of TRARG1 are phosphorylated (Fig. S3A-B), suggesting that membrane localization is required for TRARG1 phosphorylation. Together, these data suggest that TRARG1 phosphorylation does not regulate localization, but rather TRARG1 phosphorylation is dependent on TRARG1 membrane-localization, and appears to be specific to PM-localized TRARG1 in adipocytes (Fig. S1A and S3F) [5].

Since the GSK3 motif we have identified falls within a heavily phosphorylated region of TRARG1, and multisite phosphorylation often occurs at protein binding interfaces [25, 27], we next tested whether insulin-regulated and GSK3mediated phosphorylation affects TRARG1 PPIs. Serum-starved 3T3-L1 adipocytes were treated with a vehicle control (DMSO control; basal), a GSK3 inhibitor (LY2090314; GSK3i) or insulin. TRARG1 was immunoprecipitated using an optimized lysis protocol to solubilize TRARG1 (Fig. S3C-D), with immunoblotting confirming that less phosphorylated TRARG1 was immunoprecipitated from cells treated with
GSK3i or insulin compared to basal cells (Fig. 4B). PPIs were determined by subjecting immunoprecipitates to MS analysis. We identified 76 significantly enriched candidate interactors with an adj. $\mathrm{p} \leq 0.05$ and $\log _{2}$ (fold change) $\geq 2$ under basal conditions (Fig. 4B). To specifically test whether GSK3-mediated TRARG1 phosphorylation altered TRARG1 interactors, we used GSK3i and determined the TRARG1 interactome. The degree of enrichment of 14 interactors was significantly changed in response to GSK3 inhibition compared with control cells as identified by MAD analysis (4.22.2.) (Fig. 4C), with the majority of these (11 out of 14) interactions decreased in response to GSK3 inhibition. This indicates that manipulation of GSK3-mediated TRARG1 phosphorylation can alter TRARG1 PPIs. In support of this, almost half (6) of these GSK3i-regulated interactors were regulated in the same way by insulin treatment, which also inactivates GSK3 and dephosphorylates TRARG1 (Fig. 4C-E). Among these interactors commonly regulated by GSK3i and insulin, only the binding of CLPTM1 and GM14569 to TRARG1 was increased, interactions between TRARG1 and BCL9L (Bcell CLL/lymphoma 9-like protein), PYGO2 (pygopus 2), JAGN1 (protein jagunal homolog 1) and HSD17B12 (very-long-chain 3-oxoacyl-CoA reductase) were all down-regulated (Fig. 4C-E), suggesting that these proteins bind to the heavily phosphorylated/charged region of TRARG1 and are discharged upon TRARG1 dephosphorylation. Together, these data suggest that GSK3-mediated phosphorylation regulates TRARG1 PPIs, but does not directly regulate TRARG1 subcellular localization.

\section{Functional screening of selected TRARG1 interactors}

Given the role of TRARG1 in insulin-regulated GLUT4 trafficking, we tested whether TRARG1 interactors played a role in GLUT4 translocation to the PM. All of the TRARG1 interactors identified in Fig. 4 as regulated by both GSK3i and insulin were tested, as well as other TRARG1 interactors selected based on their enrichment in immunoprecipitates and reported functions in membrane trafficking processes (Fig. 5A-B and S4A). Among these, BCL9L was the most highly enriched and most regulated interactor (Fig. 4CE); RNF20 forms a complex with RNF40 and participates in mitotic spindle assembly by monoubiquitinating the microtubule motor 
bioRxiv preprint doi: https://doi.org/10.1101/2020.12.07.415257; this version posted December 7, 2020. The copyright holder for this preprint (which was not certified by peer review) is the author/funder, who has granted bioRxiv a license to display the preprint in perpetuity. It is made available under aCC-BY 4.0 International license.

protein EG5 [28]; IFITM3 shares the same membrane topology and CD225/IFITM domain with TRARG1 [29, 30]; ATAT1 and SPECC1L are involved in microtubule stabilization $[31,32]$; STEAP3 is a NADPH oxidoreductase involved in transferrin-dependent iron uptake [33, 34]. Genes were knocked down by siRNA and GLUT4 translocation to the PM was measured in adipocytes expressing a GLUT4 reporter construct with an HA-tag in the first exofacial loop and mRuby 3 at the cytosolic $\mathrm{C}$-terminal tail (HA-GLUT4-mRuby3). Surface HA-GLUT4mRuby3 was increased with insulin treatment compared to untreated cells as indicated by the increase of HA signal (Fig. 5A), and total GLUT4 as indicated by the signal of mRuby 3 was not obviously changed by the treatment (Fig. 5A). The ratio of surface versus total HA-GLUT4mRuby3 was calculated as an indicator of GLUT4 translocation to the PM. As a positive control, the knockdown of the established regulators of GLUT4 trafficking affected GLUT4 translocation as previously reported [8, 9, 35] (knockdown of $A k t 1 / 2$ or Rab10 decreased PM GLUT4, knockdown of As160 or Trarg1 trended towards an increase or a decrease of PM GLUT4, respectively, however, the significance was affected by correction for multiple comparisons in this experiment; Fig. 5B and S4A). Among the TRARG1 interactors tested, knockdown of $B c l 9 l$ significantly increased HA-GLUT4-mRuby 3 at the PM under both basal and insulin conditions, Speccll knockdown significantly increased GLUT4 reporter at the PM under $100 \mathrm{nM}$ insulin (Fig. 5B and S4A).

Since BCL9L was the most regulated interactor in response to insulin and GSK3 inhibition, we selected BCL9L for further validation. First, we confirmed Bcl $9 l$ knockdown under the conditions used for the HA-GLUT4-mRuby3 screen (72\% knockdown; Fig. 5C). Under these same conditions, Bcl9l knockdown increased ${ }^{3} \mathrm{H}-2$ deoxyglucose (2-DOG) uptake (Fig. 5D), glucose usage from the media (Fig. 5E) and lactate production (Fig. 5F); the opposite for what was observed for Trarg1 knockdown with a knockdown efficiency of $87 \%$ (Fig. 5C-F). We next used a distinct siRNA pool to target $B \operatorname{cl} 9 l(85 \%$ knockdown; Fig. S4B), and confirmed that depletion of Bcl9l increased cell surface HAGLUT4-mRuby3 and 2-DOG uptake under basal and insulin-stimulated conditions (Fig. S4C-D). These data suggest that the TRARG1-interacting protein BCL9L is a negative regulator of insulin- stimulated GLUT4 translocation and glucose transport, providing valuable insight for further studies into the regulation of insulin sensitivity and GLUT4 trafficking by TRARG1 in adipocytes.

\section{Discussion}

TRARG1 colocalizes with GLUT4 and positively regulates GLUT4 trafficking and insulin sensitivity in adipocytes. However, its mechanism of action remains unclear. Here, we have integrated TRARG1 into the insulin signaling pathway and reported that TRARG1 is a novel substrate of GSK3 and its phosphorylation is regulated through the PI3K/Akt/GSK3 axis. Further, Trarg1 knockdown blunted the potentiation of submaximal insulin-stimulated GLUT4 trafficking induced by GSK3 inhibition. Our data indicates that mouse TRARG1 is primed by a yetto-be-identified kinase at S84 promoting GSK3 phosphorylation of TRARG1 at S80, S76 and S72. This phosphorylation does not influence TRARG1 subcellular localization but plays a key role in regulating TRARG1 PPIs. Further, we identified that one of these regulated TRARG1 interactors, BCL9L, a transcriptional co-activator of $\beta$-catenin, plays a role in regulating plasma membrane GLUT4 content.

The regulation of glucose transport by insulin occurs through the integration of signaling and trafficking processes that cooperate in bringing GLUT4 to the cell surface [36]. However, the precise points of intersection between upstream signaling via Akt and the distal GLUT4 trafficking machinery are not fully resolved. Although there are conflicting reports, previous studies suggested that the Akt substrate GSK3 may play a role in glucose uptake and GLUT4 traffic [15, 20-22]. However, the exact GSK3 substrates involved in this effect on GLUT4 are not yet clear. Here we corroborate previous reports by showing that acute pharmacological inhibition of GSK3 potentiated insulin-stimulated GLUT4 translocation (Fig. 2K). This effect was impaired by Trargl knockdown at a submaximal insulin dose (Fig. 2L), suggesting that TRARG1 (de)phosphorylation may provide a link between GSK3 activity and GLUT4 trafficking. However, Trarg1 knockdown did not prevent GSK3imediated increase in PM GLUT4 in cells treated with a maximal dose of insulin (Fig. 2L), highlighting the need for further work to 
bioRxiv preprint doi: https://doi.org/10.1101/2020.12.07.415257; this version posted December 7, 2020. The copyright holder for this preprint (which was not certified by peer review) is the author/funder, who has granted bioRxiv a license to display the preprint in perpetuity. It is made available under aCC-BY 4.0 International license.

understand the exact role of the GSK3-mediated TRARG1 phosphorylation in GLUT4 trafficking.

We initially observed that endogenous phosphorylated TRARG1 was enriched at the PM but not in LDM or HDM fractions [5] (Fig. S1A), suggesting that GSK3 may phosphorylate TRARG1 at this site. This is supported by our finding that mutation of TRARG1 phosphosites did not alter TRARG1 localization (Fig. 4A), suggesting that localization may impart phosphorylation status, and not vice versa. Indeed, GSK3 has been reported to localize to the PM at the early stage of WNT signaling activation [37] and our subcellular fractionation analysis showed that GSK3 was mainly localized to the LDM and cytosol, with a small proportion of GSK3 localized to the PM (Fig. S3F). Further supporting a role for TRARG1 localization in promoting phosphorylation, the TRARG1 mutant (del_101-173), which abolishes its $\mathrm{PM}$ /membrane localization, was not phosphorylated in HEK-293E cells as indicated by the lack of apparent higher molecular weight bands (Fig. S3A-B). Of note, a TRARG1 mutant (del_129-173) localized to intracellular membranes was still phosphorylated in HEK293E cells, suggesting that there may be differential control of TRARG1 phosphorylation between HEK-293E cells and adipocytes. Future studies on TRARG1 mutants in adipocytes may help to shed light on this. Nevertheless, our data suggest that TRARG1 phosphorylation on residues between 70 and 90 does not affect TRARG1 localization, but rather TRARG1 phosphorylation is localization-dependent.

TRARG1 amino acid sequence alignment across multiple species revealed a highly conserved phosphosite-rich region between residues 70 and 90 in the murine TRARG1 N-terminal cytosolic region, with conservation as far back as Callorhincus milii, the earliest extant species in which TRARG1 is found. Using changes in phosphosite abundance in response to insulin and GSK3 inhibition and phosphosite mutagenesis, we identified a GSK3-priming site (mouse S84) and GSK3 phosphorylation sites (mouse S72, S76 and S80). The S76 and S80 GSK3 target sites were highly conserved, at least within placental mammals (Fig. 3F), suggesting an important role of regulated phosphorylation for TRARG1 function. One key difference within mammals is the presence of a third GSK3 site in rodents (mouse S72), that is almost exclusively proline in human and other mammals (Supplemental table S2). Nevertheless, the loss of phosphorylation upon mutation of the S87 priming site in human TRARG1 (Fig. 4G) and loss of endogenous human TRARG1 phosphorylation in SGBS adipocytes treated with a GSK3 inhibitor (Fig. S2C) suggest that our findings using mouse TRARG1 are translatable to human TRARG1.

Hotspots of protein phosphorylation such as those we have identified in TRARG1 have been implicated in modulating PPIs [25], and may serve as an integration point for multiple signaling pathways [38]. Indeed, in general there were multiple apparent higher molecular weight immuno-reactive TRARG1 bands observed in both HEK cells and adipocytes (Fig. S1D, 2D-E and $2 \mathrm{H}$ ), suggesting distinct phospho-species. These TRARG1 phospho-species depend on GSK3 since inhibition or knockdown of GSK3 almost completely ablated all apparent higher molecular weight forms of TRARG1 (Fig. 2E and $\mathrm{H})$. This suggests the multiple apparent higher molecular weight bands result from phosphorylation at some of the GSK3 sites, but not others (e.g. S80 and S76, but not S72), or that GSK3-mediated phosphorylation of TRARG1 leads to phosphorylation at alternate sites not targeted directly by GSK3 (Fig. 1B and 3A). Understanding the role that distinct TRARG1 phospho-species play in TRARG1 function will be the focus of future studies. To begin to address this, we assessed how GSK3 regulates TRARG1 PPIs using insulin or a GSK3 inhibitor to decrease TRARG1 phosphorylation. This analysis found several TRARG1 interactions commonly regulated by both GSK3 inhibition and insulin. Notably, most of the changes in these commonlyregulated PPIs were decreased with treatments compared to control cells, suggesting that they may bind to the phosphoserine/threonine motif (residues 70-90 for murine TRARG1), which has been shown to bind to various protein domains [39]. The key role of phosphorylation in modulating TRARG1 interactions also raises several questions about the regulation of TRARG1 phosphorylation status including: 1) the identity of the priming kinase for subsequent GSK3 activity; 2) whether other kinases can also target this region of TRARG1 in addition to GSK3; and 3) which phosphatase dephosphorylates TRARG1. Our analysis of the TRARG1 interactome may have revealed an answer to this final question, since we have identified PPP1R12A and PPP1CB, the 
bioRxiv preprint doi: https://doi.org/10.1101/2020.12.07.415257; this version posted December 7, 2020. The copyright holder for this preprint (which was not certified by peer review) is the author/funder, who has granted bioRxiv a license to display the preprint in perpetuity. It is made available under aCC-BY 4.0 International license.

regulatory and catalytic subunit of protein phosphatase 1, as TRARG1 interactors (Supplemental table S3). A possible role for protein phosphatase 1 in TRARG1 dephosphorylation is supported by our finding that inhibitors of protein phosphatase 1 and $2 \mathrm{~A}$ increased TRARG1 phosphorylation (Fig. 1E-F). Further studies into the regulation of TRARG1 phosphorylation may reveal how different signaling inputs alter TRARG1 function through the regulation of PPIs.

TRARG1 dephosphorylation altered its binding partners (Fig. 4C-E). Since TRARG1 plays a role in regulating insulin-stimulated GLUT4 translocation and glucose uptake $[5,6]$, we screened TRARG1 interactors for a role in GLUT4 trafficking. This identified that knockdown of Bcl9l and Speccll altered GLUT4 PM abundance. BCL9L, along with PYGO2 and BCL9, are transcriptional co-activators for $\beta$ catenin [40], which has a reported role in insulin sensitivity through both WNT signaling pathway $[41,42]$ and direct regulation of GLUT4 trafficking in muscle cells via cytoskeleton remodelling [43]. PYGO2 was also found to interact with TRARG1 and this interaction was regulated by insulin-stimulation and GSK3 inhibition, but Pygo2 knockdown did not influence GLUT4 trafficking, nor did Bcl9 knockdown (Fig. 5B). Indeed, although BCL9L (and PYGO2, BCL9) is reported to be predominantly nuclear localized, consistent with its role as a transcriptional co-activator, it is also present in the cytoplasm of ameloblasts during tooth development and supports the secretion of enamel components [44]. Bcl9l knockdown increased PM GLUT4 under both unstimulated and stimulated conditions (Fig. 5B and S4A), suggesting that BCL9L is a negative regulator of GLUT4 translocation to the PM or a positive regulator of GLUT4 endocytosis. Further studies are required to elucidate the biochemistry underlying the TRARG1-BCL9L interaction, how TRARG1 and BCL9L cooperate to regulate GLUT4 trafficking, and whether this involves a role for the $\beta$-catenin complex.

In summary, we have integrated the trafficking regulator of GLUT4-1, TRARG1, into the insulin signaling network via GSK3 and provided insights into how phosphorylation of TRARG1 by GSK3 regulates GLUT4 trafficking through its interacting partners. This provides strong basis for future studies into the exact mechanisms by which TRARG1 and its interactors regulate GLUT4 trafficking and insulin sensitivity in adipocytes.

\section{Experimental procedures}

\section{Molecular Cloning}

pcDNA3.1-HA-TRARG1, pcDNA3.1-TRARG1 and pcDNA3.1-HA-TRARG1-eGFP constructs were generated as previously described [26]. HATRARG1 sequences containing 7 (S74, S76, S79, S84, S85, T88, S90) or 12 (S70, S72, S74, S76, S79, S80, S84, S85, T88, T89, S90, Y91) $\mathrm{Ser} / \mathrm{Thr} / \mathrm{Tyr}$ to Ala point mutations and 7 or 11 (S70, S72, S74, S76, S79, S80, S84, S85, T88, T89, S90) Ser/Thr to Glu point mutations were generated from pcDNA3.1-HA-TRARG1, using Gibson assembly cloning [45, 46]. These constructs were designated as pcDNA3.1-HATRARG1-7A, pcDNA3.1-HA-TRARG1-12A, pcDNA3.1-HA-TRARG1-7E and pcDNA3.1HA-TRARG1-11E, respectively. These sequences were then shuttled into pBABE-puro vector using Gibson assembly cloning. Using overlap-extension site-directed mutagenesis [47], we mutated all the Cys in HA-TRARG1 sequence into Ser, which resulted in the construct pcDNA3.1-HA-TRARG1-C-S. Using Gibson assembly cloning, we mutated the first four Lys at the N-terminus of HA-TRARG1 into Arg, followed by the mutagenesis of the last three Lys into Arg using overlap-extension site-directed mutagenesis, the resulting construct was designated as pcDNA3.1-HA-TRARG1-K-R. TRARG1 truncation mutants pcDNA3.1-HATRARG1-del_129-173-eGFP and pcDNA3.1HA-TRARG1-del_101-173-eGFP were generated as previously described [26]. Residues 101-127 were deleted from pcDNA3.1-HATRARG1-eGFP to yield the plasmid pcDNA3.1HA-TRARG1-del_101-127-eGFP, using overlap-extension ${ }^{-}$site-directed mutagenesis. pcDNA3.1-TRARG1-del_101-127, pcDNA3.1TRARG1-del_129-173, and pcDNA3.1TRARG1-del_101-173 were generated from pcDNA3.1-TRARG1 by deleting residues 101$127,129-173$, or 101-173, respectively, using overlap-extension site-directed mutagenesis. Primer sequences are available upon request. Constructs were confirmed by Sanger sequencing.

\section{Cell culture and transfection}


bioRxiv preprint doi: https://doi.org/10.1101/2020.12.07.415257; this version posted December 7, 2020. The copyright holder for this preprint (which was not certified by peer review) is the author/funder, who has granted bioRxiv a license to display the preprint in perpetuity. It is made available under aCC-BY 4.0 International license.

3T3-L1 fibroblasts and HEK-293E/HeLa cells were maintained in Dulbecco's modified Eagle's medium (DMEM) supplemented with $10 \%$ fetal bovine serum (FBS) and GlutaMAX (Life Technologies, Inc.) (DMEM/FBS medium) at $37{ }^{\circ} \mathrm{C}$ with $10 \% \mathrm{CO}_{2}$. L6 myoblasts were grown in $\alpha$-MEM supplemented with $10 \%$ FBS and GlutaMAX at $37{ }^{\circ} \mathrm{C}$ and $10 \% \mathrm{CO}_{2}$. For 3T3-L1 stable cell lines, fibroblasts were transduced with pBABE-puro retrovirus (empty vector control), or retrovirus with the constructs of interest in pBABE-puro vector. Puromycin $(2 \mu \mathrm{g} / \mathrm{mL})$ was used to select for transduced cells. 3T3-L1 fibroblasts were cultured and differentiated into adipocytes as described previously [5], and used for experiments between day 9 and 12 after the initiation of differentiation. HEK-293E/HeLa cells were transfected with indicated constructs 2 days before experiments using lipofectamine2000 (Thermo Scientific), according to the manufacturer's instructions. Myoblasts at $80 \%$ confluence were differentiated into myotubes by replacing $10 \%$ FBS with $2 \%$ FBS. Myotubes were used in experiments at between 6-8 days post-differentiation. SGBS cells were cultured and differentiated as previously reported [48, 49], and used for experiments 12-14 days after initiation of differentiation. SGBS adipocytes were incubated in growth-factor free media for $4 \mathrm{~h}$ prior to addition of insulin or GSK3 inhibitors.

\section{Cell fractionation}

3T3-L1 adipocytes were washed with ice-cold PBS and harvested in ice-cold HES-I buffer (20 mM HEPES, $\mathrm{pH}$ 7.4, $1 \mathrm{mM}$ EDTA, $250 \mathrm{mM}$ sucrose containing protease inhibitors mixture (Roche Applied Science)). All subsequent steps were carried out at $4{ }^{\circ} \mathrm{C}$. Cells were fractionated as previously described [26]. Briefly, cells were homogenized by passing through a 22-gauge needle 5 times and a 27-gauge needle 10 times prior to centrifugation at $500 \mathrm{x} \mathrm{g}$ for $10 \mathrm{~min}$ to pellet cell debris. The supernatant was centrifuged at $13,500 \times \mathrm{g}$ for $12 \mathrm{~min}$. The pellet contained the PM and mitochondria/nuclei $(\mathrm{M} / \mathrm{N})$. The supernatant consisted of cytosol, low density microsome (LDM), and high density microsome (HDM). This supernatant was centrifuged at $21,170 \mathrm{x} g$ for $17 \mathrm{~min}$ to pellet the HDM fraction. The supernatant was again centrifuged at 235,200 $\mathrm{x} g$ for $75 \mathrm{~min}$ to obtain the LDM fraction (pellet). The pellet containing $\mathrm{PM}$ and $\mathrm{M} / \mathrm{N}$ was referred to as PM fraction in this study as TRARG1 is not enriched in $\mathrm{M} / \mathrm{N}$ fractions (data not shown).

\section{Lambda protein phosphatase assay in 3T3-L1 adipocytes/HEK-293E and fat tissues}

Cells were washed with PBS twice and lysed in $1 \%(\mathrm{v} / \mathrm{v})$ Triton X-100 in PBS and then solubilized by passing through a 22 -gauge needle 3 times and a 27-gauge needle 3 times prior to centrifugation at $12,000 \times \mathrm{g}$ for $15 \mathrm{~min}$. In the case of fat tissues, subcutaneous/epidydimal white adipose tissue or brown adipose tissue was excised from mice and lysed in $1 \%(\mathrm{v} / \mathrm{v})$ Triton $\mathrm{X}-100$ in PBS. Tissues were lysed and homogenized by sonication (Gallay Scientific) prior to centrifugation at $13,000 \mathrm{x}$ g for $15 \mathrm{~min}$. The supernatant was transferred to a clean tube and protein concentration determined by bicinchoninic acid (BCA) assay (Thermo Scientific). $100 \mu \mathrm{g}$ of tissue or cell lysates were treated with or without $2 \mu \mathrm{L}$ Lambda Protein Phosphatase (LPP) (New England Biolabs) at 30 ${ }^{\circ} \mathrm{C}$ for $15 \mathrm{~min}$ (30 min for HEK-293E lysate) in a reaction volume of $50 \mu \mathrm{L}$. Starting material without $15 \mathrm{~min}$ (30 $\mathrm{min}$ for HEK-293E lysate) incubation at $30{ }^{\circ} \mathrm{C}$ was included as a control. Reaction was terminated by the addition of SDS $(1 \%(\mathrm{w} / \mathrm{v})$ final concentration), and the reaction mixture was incubated at $95{ }^{\circ} \mathrm{C}$ for a further 10 min. Loading sample buffer (LSB) with TCEP was added to the samples, which were then incubated at $65{ }^{\circ} \mathrm{C}$ for $5 \mathrm{~min}$, separated by SDSPAGE, and analyzed by immunoblotting.

\section{Phosphatase inhibitors assay and kinase inhibitors assay in 3T3-L1 adipocytes}

3T3-L1 fibroblasts were seeded in 12-well plates and differentiated into adipocytes. Cells were used for experiments on day 10 postdifferentiation. For phosphatase inhibitors assay, adipocytes were treated with $100 \mathrm{nM}$ calyculin A, $1 \mu \mathrm{M}$ okadaic acid or DMSO in DMEM/FBS medium for $60 \mathrm{~min}$. For kinase inhibitors assay, cells were serum-starved in basal DMEM media (DMEM, GlutaMAX, 0.2\% (w/v) bovine serum albumin (BSA, Bovostar, Bovogen)) for $2 \mathrm{~h}$ prior to treatment with kinase inhibitors $(100 \mathrm{nM}$ wortmannin, $10 \mu \mathrm{M}$ MK-2206, $100 \mu \mathrm{nM}$ rapamycin or $1 \mu \mathrm{M}$ GDC-0994) for $20 \mathrm{~min}$ followed by $100 \mathrm{nM}$ insulin treatment for $20 \mathrm{~min}$. Cells were transferred onto ice and washed with ice-cold PBS twice, followed by lysis with $1 \%$ $(\mathrm{w} / \mathrm{v})$ SDS in PBS-containing protease inhibitors 
bioRxiv preprint doi: https://doi.org/10.1101/2020.12.07.415257; this version posted December 7, 2020. The copyright holder for this preprint (which was not certified by peer review) is the author/funder, who has granted bioRxiv a license to display the preprint in perpetuity. It is made available under aCC-BY 4.0 International license.

mixture (Roche Applied Science) and phosphatases inhibitors mixture $(1 \mathrm{mM}$ sodium pyrophosphate, $2 \mathrm{mM}$ sodium orthovanadate, 10 $\mathrm{mM}$ sodium fluoride). Cell lysates were sonicated at $90 \%$ intensity for $12 \mathrm{~s}$ and centrifuged at $13,000 \mathrm{x}$ g. Protein concentration of the supernatant was determined by BCA assay. LSB was added to the samples, which were then incubated at $65{ }^{\circ} \mathrm{C}$ for $5 \mathrm{~min}$, separated by SDSPAGE, and analyzed by immunoblotting.

\section{GSK3 inhibitors treatment in adipose tissue explants}

Epididymal fat depots were excised from mice, transferred immediately to warm DMEM/ $2 \%$ $\mathrm{BSA} / 20 \mathrm{mM}$ HEPES, $\mathrm{pH} 7.4$, and minced into fine pieces. Explants were washed 3 times and incubated in DMEM $/ 2 \% \mathrm{BSA} / 20 \mathrm{mM}$ HEPES, $\mathrm{pH} 7.4$ for $2 \mathrm{~h}$. Explants were then aliquoted and treated with $10 \mathrm{nM}$ insulin, $500 \mathrm{nM}$ LY 2090314 or DMSO for $30 \mathrm{~min}$ at $37^{\circ} \mathrm{C}$. Treatment was terminated with three rapid washes in ice-cold PBS, after which the cells were solubilized in RIPA buffer (50 mM Tris-HCl, $\mathrm{pH} 7.5,150 \mathrm{mM}$ $\mathrm{NaCl}, 1 \%(\mathrm{v} / \mathrm{v})$ Triton X-100, 0.5\% (w/v) sodium deoxycholate, $0.1 \%(\mathrm{w} / \mathrm{v})$ SDS, $1 \mathrm{mM}$ EDTA, protease inhibitors mixture). Samples were then subjected to sonication prior to spin at $20,000 \mathrm{x} \mathrm{g}$ for $15 \mathrm{~min}$ at $4{ }^{\circ} \mathrm{C}$. The supernatant was transferred to a clean tube and protein concentrations were determined using BCA assay. LSB with TCEP was added to the samples, which were then incubated at $65^{\circ} \mathrm{C}$ for $5 \mathrm{~min}$, separated by SDS-PAGE, and analyzed by immunoblotting.

\section{esiRNA-mediated GSK3 Knockdown}

900 nM esiRNA (Sigma, EMU170441, EMU059761) was added to $100 \mu \mathrm{L}$ Opti-MEM medium with $7.5 \mu \mathrm{L}$ transfection reagent TransIT-X2 (Mirus Bio), mixed and incubated at room temperature (RT) for $30 \mathrm{~min}$. Adipocytes at 6 days post-differentiation were washed once with PBS, trypsinized with $5 \mathrm{x}$ trypsin-EDTA (Life Technologies, Inc.) at $37^{\circ} \mathrm{C}$, resuspended in DMEM/FBS medium and then centrifuged at 200 $\mathrm{x} g$ for $5 \mathrm{~min}$. The supernatant was removed, and pelleted cells were resuspended in DMEM/FBS medium of the same volume as that of the media where the cells were previously cultured (e.g. 1 $\mathrm{mL}$ of media for the cells from one well of a 12well plate). $900 \mu \mathrm{L}$ of cell suspension was then reseeded onto one well of a Matrigel-coated 12well plate. $100 \mu \mathrm{L}$ of esiRNA or luciferase control mixture was added into per well of a 12well plate. Cells and esiRNA mixture were mixed and returned to incubator and used in experiments $72 \mathrm{~h}$ following esiRNA knockdown.

\section{Immunoprecipitation of TRARG1 and in vitro kinase assay}

3T3-L1 adipocytes at day 10 post-differentiation were serum-starved in basal DMEM media containing $100 \mathrm{nM}$ LY2090314 for 2 h. Cells were then transferred to ice, washed thrice with ice-cold PBS and harvested in lysis buffer $(1 \%$ NP 40, 5\% glycerol, 50 mM Tris- $\mathrm{HCl}$, pH 7.4, $150 \mathrm{mM} \mathrm{NaCl}$ ) containing protease inhibitors mixture and phosphatase inhibitors mixture. Cells were lysed by passing through a 22-gauge needle six times, followed by six times through a 27gauge needle. Lysates were solubilized on ice for $20 \mathrm{~min}$ and then centrifuged at $20,000 \mathrm{x}$ g for 20 $\min$ at $4{ }^{\circ} \mathrm{C}$ to remove insoluble material. The protein concentration of the supernatant was quantified by BCA assay following the manufacturer's protocol. For each sample, $2.5 \mathrm{mg}$ protein was combined with $5 \mu \mathrm{L}$ of anti-TRARG1 mouse monoclonal antibody $(200 \mu \mathrm{g} / \mathrm{mL})$ (sc$377025)$ or $2.5 \mu \mathrm{L}$ of mouse anti-IgG $(400 \mu \mathrm{g} / \mathrm{mL})$ in a final volume of $500 \mu \mathrm{L}$ and incubated with rotation at $4{ }^{\circ} \mathrm{C}$ for $1 \mathrm{~h}$. Magnetic Dynabeads (Life Technologies, 10004D) were washed once with ice-cold PBS, once with lysis buffer and then resuspended in lysis buffer. $50 \mu \mathrm{L}$ of magnetic beads were then added into each immunoprecipitation (IP) tube followed by incubation with rotation at $4{ }^{\circ} \mathrm{C}$ for $1 \mathrm{~h}$. Beads were then separated from flow-through by magnetic capture and resuspended and washed twice with lysis buffer, followed by two washes with ice-cold PBS. The residual liquid was removed from the final wash. The TRARG1-IP tubes and IgG control tube were incubated with either kinase assay buffer $(20 \mathrm{mM}$ Tris- $\mathrm{HCl}, \mathrm{pH}$ 7.4, $10 \mathrm{mM} \mathrm{MgCl}$, $1 \mathrm{mM}$ DTT, $1 \mathrm{mM}$ sodium pyrophosphate and $10 \mathrm{mM}$ sodium fluoride, 10 mM glycerol-2-phosphate, $2 \mathrm{mM} \mathrm{ATP)} \mathrm{or} \mathrm{kinase}$ assay buffer containing either $1 \mu \mathrm{g}$ GSK $3 \alpha$ or 1 $\mu \mathrm{g}$ GSK3 $\beta$ in a final volume of $30 \mu \mathrm{L}$ at $37{ }^{\circ} \mathrm{C}$ for $1 \mathrm{~h}$. Following the incubation, reactions were terminated, and proteins were eluted by the addition of $30 \mu \mathrm{L} 2 \mathrm{X} \mathrm{LSB}$ and incubation at $65^{\circ} \mathrm{C}$ for $5 \mathrm{~min}$. Samples were then centrifuged at $20,000 \mathrm{x}$ g for $2 \mathrm{~min}$ at RT and stored at $-20^{\circ} \mathrm{C}$.

\section{Polymorphism analysis}


TRARG1 sequences were extracted from Ensembl (release 99) and aligned by Multiple sequence Alignment using Fast Fourier Transform (MAFFT) version 7 [50]. Analysis of the number of polymorphisms at each residue was conducted across 64 unique placental mammalian species (represented by 69 genomes including different subspecies/sexes of the same species). The results of this analysis between residues 6791 of mouse TRARG1 are presented in Fig. 3F and the full analysis in Supplemental table S2.

\section{Immunofluorescence confocal microscopy}

3T3-L1 adipocytes stably expressing HATRARG1, HA-TRARG1-7A or HA-TRARG17E were re-plated onto Matrigel-coated (Becton Dickinson) glass coverslips on day 6 after initiation of differentiation and processed for immunofluorescence microscopy on day 10 . Cells were washed with PBS and fixed with 4\% $(\mathrm{v} / \mathrm{v})$ paraformaldehyde (PFA) for $20 \mathrm{~min}$ at RT. Cells were blocked and permeabilized with $2 \%$ $(\mathrm{w} / \mathrm{v})$ BSA and $0.1 \%(\mathrm{w} / \mathrm{v})$ saponin in PBS followed by incubation with a mixture of rabbit anti-HA antibody (CST, C29F4) and anti-GLUT4 $1 \mathrm{~F} 8$ antibody at RT for $45 \mathrm{~min}$. Cells were washed five times with $2 \%(\mathrm{w} / \mathrm{v})$ BSA and $0.1 \%$ $(\mathrm{w} / \mathrm{v})$ saponin in PBS. Anti-mouse Alexa-555conjugated secondary antibody (Life Technologies) and anti-rabbit Alexa-488conjugated secondary antibody (Life Technologies) were used to detect primary antibodies. DAPI was used to visualize nuclei. Samples were mounted in Immuno-Fluore Mounting Medium. For HEK-293E cells expressing HA-TRARG1 truncation mutants, cells were re-plated onto Matrigel-coated glass coverslips in 12-well plate $24 \mathrm{~h}$ after transfection, allowed to adhere overnight. Samples were prepared as described above, but without antibody incubations. Optical sections were obtained using Nikon C2 Confocal microscope using a Plan Apo VC 60X WI DIC N2 (NA=1.2 $\mathrm{WD}=270 \mu \mathrm{m})$ objective. Images were acquired using NIS Elements software. All images were processed using Fiji [51].

siRNA-mediated gene knockdown for HAGLUT4-mRuby3 translocation assay, ${ }^{3} \mathrm{H}-2-$ deoxyglucose uptake assay and media glucose consumption/lactate production assay
$47 \mu \mathrm{L}$ of OptiMEM, $2 \mu \mathrm{L}$ of TransIT-X2 and 1 $\mu \mathrm{L}$ of siRNA $(25 \mu \mathrm{M}$, ON TARGETplus or siGenome, Dharmacon, final concentration 500 $\mathrm{nM}$ ) were mixed and incubated for $30 \mathrm{~min}$ at RT. On day 6 post-differentiation, 3T3-L1 adipocytes grown in a 6-well plate (stably expressing HAGLUT4-mRuby3 where appropriate) were washed once with PBS, trypsinized with 5X trypsin-EDTA at $37{ }^{\circ} \mathrm{C}$, resuspended in DMEM/FBS medium and centrifuged at $200 \mathrm{x} \mathrm{g}$ for $5 \mathrm{~min}$. The supernatant was removed, and pelleted cells were resuspended in $13.5 \mathrm{~mL}$ DMEM/FBS. $450 \mu \mathrm{L}$ of cell suspension was added into the mixture of OptiMEM/TransIT$\mathrm{X} 2 /$ siRNA and mixed well (final concentration of siRNA was $50 \mathrm{nM}$ ). All $500 \mu \mathrm{L}$ of the cell suspension plus OptiMEM/TransIT-X2/siRNA was added to one well of a Matrigel-coated 24well plate, or $84 \mu \mathrm{L}$ of the cell suspension plus OptiMEM/TransIT-X2/siRNA was aliquoted into each well of the Matrigel-coated 96-well plate. Cells were refed with DMEM/FBS medium $24 \mathrm{~h}$ post transfection and used for HA-GLUT4mRuby3 assay $72 \mathrm{~h}$ post transfection.

For Fig. 5B-5F and S4A, ON TARGETplus siRNA pools (Dharmacon) were used to knockdown Akt1 (L-040709-00), Akt2 (L040782-00), Rab10 (L-040862-01), Trarg1 (L057822-01) Bcl9l (L-042159-01), Clptm1 (L049802-01), Gm14569 (L-173034-00), Hsd17b 12 (L-060708-01), Pygo2 (L-049590-01), Rnf20 (L-041733-01), Rnf40 (L-059014-01), Bcl9 (L-049660-01), Steap3 (L-046923-01), Ifitm 3 (L-056653-01), Jagn1 (L-049015-01), Dyxlc1 (L-060904-01), Ano6 (L-065392-01), Atat1 (L-050973-02), and Specc1l (L-058993-01) in comparison to a non-targeting pool (D001810-10). For Fig. S4B-D, siGenome siRNA pools (Dharmacon) were used to knockdown Bcl9l (M-042159-01) in comparison to a nontargeting pool (D-001206-13).

\section{Endogenous GLUT4 and HA-GLUT4- mRuby3 translocation assays}

Cells were washed once with PBS and once with basal DMEM media prior to incubation in basal DMEM media for $2 \mathrm{~h}$ in a $\mathrm{CO}_{2}$ incubator. Cells were stimulated with $0.5 \mathrm{nM}$ or $100 \mathrm{nM}$ insulin for $20 \mathrm{~min}$. For endogenous GLUT4 translocation assay (Fig. 2K-L), cells were serum-starved in the presence or absence of DMSO (control) or GSK3 inhibitor prior to $0.5 \mathrm{nM}$ or $100 \mathrm{nM}$ insulin 
stimulation for $20 \mathrm{~min}$. After the stimulation, cells were washed by gently dunking the 96-well plates 12 times in a beaker containing ice cold PBS (all the subsequent PBS washes were performed using this method). Plates were placed on ice and residual PBS was removed with multichannel pipette. Cells were fixed with $4 \%$ PFA for $5 \mathrm{~min}$ on ice, and $20 \mathrm{~min}$ at RT and PFA was replaced with $50 \mathrm{mM}$ glycine in PBS followed by incubation for $15 \mathrm{~min}$. After dunking the plates 6 times in RT PBS, residual PBS was removed and cells were blocked with 5\% Normal Swine Serum (NSS, Dako, X0901) in PBS for 20 $\mathrm{min}$. After removing all the blocking media, cells were incubated with human anti-GLUT4 antibody (LM048; [52]) (Integral Molecular, PA, USA) or mouse anti-HA (1:500, Covance, MMS101R) antibody in 2\% NSS in PBS for $1 \mathrm{~h}$ to detect GLUT4 or HA-GLUT4-mRuby3 present at the PM. After 12 washes in RT PBS, residual PBS was removed and cells were incubated with antihuman Alexa-488 (for endogenous GLUT4 assays) or anti-mouse Alexa-488 or Alexa-647 secondary antibody (for HA-GLUT4-mRuby3 assays) (Life Technologies) and Hoechst 33342 (Life Technologies) in 2\% NSS in PBS for $1 \mathrm{~h}$. Cells were washed 12 times in RT PBS and stored in PBS containing $2.5 \%$ DABCO, $10 \%$ glycerol, pH 8.5 and imaged on the Perkin Elmer Opera Phenix High Content Screening System. Imaging data were analyzed using Harmony Software supplied with the imaging system. For endogenous GLUT4 translocation, surface GLUT4 was normalized to nuclear DNA (Hoechst 33342 signal). For HA-GLUT4mRuby 3 translocation assay, surface GLUT4 was indicated by the HA signal and total GLUT4 was indicated by the mRuby 3 signal. The HAGLUT4-mRuby3 translocation screening of insulin/GSK3i-regulated TRARG1 interactors and non-insulin/GSK3i-regulated interactors were performed in two standalone studies and presented as separate figures (Fig. 5B, S4A).

\section{${ }^{3} \mathrm{H}$-2-deoxyglucose uptake assay}

Following siRNA-mediated knockdown, adipocytes were serum-starved for $2 \mathrm{~h}$ in basal DMEM media, cells were washed three times and incubated in pre-warmed Krebs-Ringer phosphate buffer containing $0.2 \%$ BSA (KRP buffer; $0.6 \mathrm{mM} \mathrm{Na}_{2} \mathrm{HPO}_{4}, 0.4 \mathrm{mM} \mathrm{NaH}_{2} \mathrm{PO}_{4}, 120$ $\mathrm{mM} \mathrm{NaCl}, 6 \mathrm{mM} \mathrm{KCl}, 1 \mathrm{mM} \mathrm{CaCl} 2,1.2 \mathrm{mM}$ $\mathrm{MgSO}_{4}$ and $12.5 \mathrm{mM}$ HEPES (pH 7.4)) at $37^{\circ} \mathrm{C}$. Cells were stimulated with $0.5 \mathrm{nM}$ or $100 \mathrm{nM}$ insulin for $20 \mathrm{~min}$. To determine non-specific glucose uptake, $25 \mu \mathrm{M}$ cytochalasin B (ethanol, Sigma Aldrich) was added to the wells before addition of ${ }^{3} \mathrm{H}-2$-deoxyglucose (2-DOG) (PerkinElmer). 2-DOG $(0.25 \mu \mathrm{Ci}, 50 \mu \mathrm{M})$ was added to cells during the final $5 \mathrm{~min}$ to measure steady-state 2-DOG uptake rates. Following three washes with ice-cold PBS, cells were solubilized in PBS containing 1\% (v/v) Triton X-100 and subjected to three freeze-thaw cycles. Tracer uptake was quantified by liquid scintillation counting and data normalized for DNA content, measured as previously described except using Sybr-green (Life Technologies) instead of Hoechst-33342 [53].

\section{Media glucose consumption/lactate production assay}

Following siRNA-mediated knockdown, media was collected from wells and analyzed for glucose and lactate concentrations after $48 \mathrm{~h}$. Naïve media was used as a baseline. Media glucose was measured using an adaption of the hexokinase-glucose-6-phosphate dehydrogenase method described by Kunst et al [54] (Siemens Healthcare (product code DF30)). Media lactate was measured by monitoring absorbance at 340 $\mathrm{nm}$ due to NADH production as L-lactate is oxidized to pyruvate by lactate dehydrogenase (Siemens Healthcare (product code DF16)). All data presented as nmol glucose consumed or lactate produced normalized to DNA content, measured as previously described except using Sybr-green (Life Technologies) instead of Hoechst-33342 [53].

\section{Sample preparation and real-time quantitative-PCR assays}

For Fig. 5C and S4B, total RNA was extracted from cells using QIAshredder and RNeasy kits (Qiagen). To remove any DNA, the extracts were incubated with DNAse buffer (Promega) and residual DNAse was subsequently inactivated with DNAse stop solution (Promega). cDNA synthesis was performed using LunaScript RT SuperMix Kit (NEB). Polymerase chain reactions were carried out using TaqMan 2X Universal PCR Master Mix or SYBR Green PCR Master Mix (Thermo) on a QuantStudio ${ }^{\text {TM }} 7$ Flex RealTime PCR System (Thermo). Acidic ribosomal phosphoprotein P0 (36B4), $\beta$-actin (b-act) and 18S ribosomal RNA (18s) were used as internal controls. The following primer sets were used: 
bioRxiv preprint doi: https://doi.org/10.1101/2020.12.07.415257; this version posted December 7, 2020. The copyright holder for this preprint (which was not certified by peer review) is the author/funder, who has granted bioRxiv a license to display the preprint in perpetuity. It is made available under aCC-BY 4.0 International license.

36B4_F; AGATGCAGCAGATCCGCAT and 36B4_R; GTTCTTGCCCATCAGCACC, bact F; GCTCTGGCTCCTAGCACCAT and bact_R; GCCACCGATCCACACAGAGT, and 18s_F; CGGCTACCACATCCAAGGAA and 18s_R; GCTGGAATTACCGCGGCT, with the corresponding 18s taqman probe GAGGGCAAGTCTGGTGCCAG. The TaqMan gene expression assays (premixed primer set and probes) were used for mouse Trargl (Mm03992124_m1) and Bcl9l (Mm01143422_m1).

\section{Western blotting}

Typically, $10 \mu \mathrm{g}$ of protein was resolved by SDSPAGE, transferred to PVDF membranes and immunoblotted as previously described [5]. Antibodies detecting TRARG1 (sc-292062 or sc377025) and 14-3-3 (sc-1657) were from Santa Cruz Biotechnology. Antibodies against pHSL S660 (\#4126), pp90RSK (\#9344), p4EBP1 S65 (\#9456), pTBC1D4 T642 (\#4288S), pAkt S473 (\#4051), pAkt T308 (\#9275L), Akt (\#4685), HA (\#C29F4), GSK3 $\alpha$ (\#9338S), GSK3 $\beta$ (\#9832S), pGSK3 Ser 9/21 (\#8566S), pGS S641 (\#3891) and Caveolin1 (\#3267) were purchased from Cell Signaling Technology. Anti- $\alpha$-tubulin (\#T9026) was from Sigma-Aldrich. Antibody against TBC1D4 was produced as previously described [4].

\section{Sample preparation for MS-based PTMomics of TRARG1}

HEK-293E cells transiently expressing HATRARG1 or TRARG1 were washed three times with ice-cold PBS, lysed in RIPA buffer and homogenized prior to centrifugation at 20,000 x $\mathrm{g}, 4^{\circ} \mathrm{C}$ for $20 \mathrm{~min}$ to remove cellular debris. $2 \mathrm{mg}$ of each cell lysate was incubated with $40 \mu \mathrm{L}$ AntiHA Microbeads ( $\mu$ MACS HA Isolation Kit, Miltenyi Biotec, 130-091-122) in a volume of 500 $\mu \mathrm{L}$ for $45 \mathrm{~min}$ at $4{ }^{\circ} \mathrm{C}$ with rotation. Microbeads were separated from flow-through by running through $\mu$ Columns (MACS, Miltenyi Biotec, 130-042-701) and three washes with RIPA buffer, followed by two washes with ice-cold PBS. Proteins were eluted in 2X LSB and applied for SDS-PAGE. Gels were stained with SyproRuby protein gel stain (Life Technologies) according to the manufacturer's instructions and imaged on a Typhoon FLA 9500 biomolecular imager (GE Healthcare). TRARG1 bands were excised for ingel digestion.
Gel fractions were washed in 50\% acetonitrile (ACN, Thermo Scientific) in $100 \mathrm{mM}$ ammonium bicarbonate $\left(\mathrm{NH}_{4} \mathrm{HCO}_{3}\right.$, Sigma) at RT for $2 \mathrm{X}$ $5 \mathrm{~min}$ at 2,000 rpm in a ThermoMixer $\mathrm{C}$ (Eppendorf). Liquid was removed, $10 \mathrm{mM}$ TCEP and $40 \mathrm{mM}$ 2-Chloroacetamide (CAA, Sigma) in $100 \mathrm{mM} \mathrm{NH}_{4} \mathrm{HCO}_{3}$ was added to the gel fractions and incubated at RT for $30 \mathrm{~min}$ at 2,000 rpm. Liquid was removed and gel pieces were dehydrated in $100 \% \mathrm{ACN}$ at RT for $5 \mathrm{~min}$ at $2,000 \mathrm{rpm}$ followed by rehydration in $100 \mathrm{mM}$ $\mathrm{NH}_{4} \mathrm{HCO}_{3}$ containing $14 \mathrm{ng} / \mu \mathrm{L}$ trypsin (Promega) on ice for $1 \mathrm{~h}$. Excess liquid was removed and 100 $\mu \mathrm{L}$ of $100 \mathrm{mM} \mathrm{NH}_{4} \mathrm{HCO}_{3}$ was added to gel slices and samples were incubated at $37{ }^{\circ} \mathrm{C}$ overnight. Peptides were solubilized by spiking in Trifluroacetic acid (TFA, Thermo Scientific) to 1\% and incubating at $37^{\circ} \mathrm{C}$ for $30 \mathrm{~min}$. To extract peptides, gel pieces were dehydrated using $100 \%$ ACN and incubated at $37^{\circ} \mathrm{C}$ for 15 min. Peptides were transferred to a new tube and dried in a vacuum concentrator (Eppendorf) and then resuspended and acidified in 1\% TFA.

Resuspended peptides were centrifuged at 21,000 $\mathrm{x} g$ for $10 \mathrm{~min}$ at $4^{\circ} \mathrm{C}$. The peptide mixture was desalted using stage-tips containing $2 \mathrm{X}$ styrene divinylbenzene-reverse phase sulfonated (SDBRPS) (Empore, 3M). StageTips were preequilibrated with addition of $100 \% \mathrm{ACN}, 30 \%$ methanol (Fisher Scientific) and 1\% TFA then $0.2 \% \mathrm{TFA}$ and $5 \% \mathrm{ACN}$ and centrifugation at $1,500 \mathrm{x} g$ for $3 \mathrm{~min}$. After loading the samples, StageTips were washed once with $99 \%$ isopropanol $/ 1 \%$ TFA and once with $0.2 \% \mathrm{TFA} / 5 \%$ ACN, then peptides were eluted from StageTips with elution buffer $(60 \%$ ACN, $5 \%$ NH4OH $(20 \%$ $\mathrm{v} / \mathrm{v}$ of a $25 \% \mathrm{NH} 4 \mathrm{OH}$ solution)). Samples were dried in a vacuum concentrator for $\sim 45 \mathrm{~min}$, at $45^{\circ} \mathrm{C}$ and resuspended in $5 \mu \mathrm{l}$ MS loading buffer $(0.3 \%$ TFA, $2 \% \mathrm{ACN})$ for MS analysis.

For phosphoproteomics analysis in 3T3-L1 adipocytes treated with GSK3 inhibitor, samples were lysed and prepared using the recently described EasyPhos method [55, 56], before being resuspended in $5 \mu \mathrm{L}$ MS loading buffer $(2 \%$ ACN, 0.3\% TFA).

\section{Sample preparation for TRARG1 interactome analysis}

3T3-L1 adipocytes were washed three thrice with ice-cold PBS and lysed in lysis buffer (1\% (w/v) 
bioRxiv preprint doi: https://doi.org/10.1101/2020.12.07.415257; this version posted December 7, 2020. The copyright holder for this preprint (which was not certified by peer review) is the author/funder, who has granted bioRxiv a license to display the preprint in perpetuity. It is made available under aCC-BY 4.0 International license.

CHAPS, $150 \mathrm{mM} \mathrm{NaCl}, 50 \mathrm{mM}$ Tris-HCl, $\mathrm{pH} 7.5$, $5 \%(\mathrm{v} / \mathrm{v})$ glycerol with protease inhibitor mixture and phosphatase inhibitors) by passing through a 22 -gauge needle 6 times, followed by a 27-gauge needle 3 times. Octyl $\beta$-D-glucopyranoside (Bog) was added into each lysate to a final concentration of $50 \mathrm{mM}$ and lysates were solubilized for $30 \mathrm{~min}$ with rotation at $4{ }^{\circ} \mathrm{C}$ prior to centrifugation at $20,000 \mathrm{x} \mathrm{g}$ for $20 \mathrm{~min}$ at $4{ }^{\circ} \mathrm{C}$ to remove lipid and cell debris. The resulting supernatants were transferred into a new tube and centrifuged at $265,100 \mathrm{x} \mathrm{g}$ for $30 \mathrm{~min} .8 \mathrm{mg}$ of each lysate was incubated with $16 \mu \mathrm{L}$ of anti-TRARG1 antibody or the same amount of IgG of the same species for $1 \mathrm{~h}$ with rotation at $4{ }^{\circ} \mathrm{C} .100 \mu \mathrm{L}$ Magnetic Dynabeads were added into each antibody-lysate mixture and incubated for $1 \mathrm{~h}$ with rotation at $4{ }^{\circ} \mathrm{C}$. Beads were washed three times with Wash Buffer I and twice with Wash Buffer II. For MS sample preparation, beads were incubated in $25 \mu \mathrm{L}$ Elution Buffer I for $30 \mathrm{~min}$ at RT, and $2 \mathrm{X} 50 \mu \mathrm{L}$ Elution Buffer II was added. Each eluate was collected into a LowBind Eppendorf tube and digested overnight at RT. Peptides were acidified by adding TFA to a final concentration of $1 \%$.

StageTips containing 2X SDB-RPS (Empore, 3M) were pre-equilibrated with addition of $100 \%$ $\mathrm{ACN}, 30 \%$ methanol (Fisher Scientific) and 1\% TFA then $0.2 \%$ TFA and $5 \% \quad \mathrm{ACN}$ and centrifugation at $1,500 \times \mathrm{g}$ for $3 \mathrm{~min}$. Peptide samples were loaded and washed with $1 \%$ TFA in isopropanol, then $0.2 \% \mathrm{TFA}$ and $5 \% \mathrm{ACN}$. Peptides were eluted from the SDB-RPS tips in $60 \% \mathrm{ACN}$ and $20 \% \mathrm{NH}_{4} \mathrm{OH}$ (25\%, HPLC grade, Sigma). Samples were dried in a vacuum concentrator for $50 \mathrm{~min}$ at $45^{\circ} \mathrm{C}$ and resuspended in $2 \% \mathrm{ACN} / 0.3 \%$ TFA for LC-MS/MS analysis.

\section{Mass spectrometry}

For TRARG1 interactome study, mass spectrometry analysis was performed on a LC/MS system including an EASY-nLC coupled to a Q Exactive HF-X mass spectrometer. Peptides were separated using a $75 \mu \mathrm{m} \times 40 \mathrm{~cm}$ column packed in-house (ReproSil Pur C18-AQ, $1.9 \mu \mathrm{m}$ particle size) with a gradient of $5-40 \%$ (EASY-nLC) buffer B ( $80 \% \mathrm{ACN} / 0.1 \% \mathrm{FA})$ over $40 \mathrm{~min}$ at $300 \mathrm{~nL} / \mathrm{min}$ with the column maintained at $60^{\circ} \mathrm{C}$ using a column oven. The mass spectrometer was operated in datadependent acquisition mode, acquiring survey scans of $3 \mathrm{e}^{6}$ ions at a resolution of 60,000 from $300-1,650 \mathrm{~m} / \mathrm{z}$. 20 of the most abundant precursors from the survey scan with charge state $>1$ and $<6$ were selected for fragmentation. Precursors were isolated with a window of 1.4 $\mathrm{m} / \mathrm{z}$ and fragmented in the HCD cell with NCE of 27. Maximum ion fill times for the MS/MS scans were $28 \mathrm{~ms}$ and target fill value was $1 \mathrm{e}^{4}$ ions. Fragment ions were analyzed with high resolution $(15,000)$ in the Orbitrap mass analyzer. Dynamic exclusion was enabled with duration 15-20 s.

For MS-based PTMomics of TRARG1, an Easy nLC-1000 UHPLC was connected to a Q Exactive mass spectrometer for mass spectrometry analysis. A $75 \mu \mathrm{m} \times 40 \mathrm{~cm}$ column packed in-house (ReproSil Pur C18-AQ, $1.9 \mu \mathrm{m}$ particle size) was used to separate the peptides prior to elution with a gradient of 5-30\% ACN containing $0.1 \%$ formic acid (FA) at $200 \mathrm{~nL} / \mathrm{min}$ over 90 min with the column temperature set at $55^{\circ} \mathrm{C}$. Peptides were analyzed with one MS scan from 300 to $1750 \mathrm{~m} / \mathrm{z}$ with a resolution of 70,000 , $3 \mathrm{e}^{6} \mathrm{AGC}$, and a max injection time (IT) of $100 \mathrm{~ms}$, followed by data-dependent MS/MS scans with HCD at a resolution of 17,500, AGC $5 \mathrm{e}^{5}$, IT $60 \mathrm{~ms}$, NCE $25 \%$, and isolation width of $2.0 \mathrm{~m} / \mathrm{z}$. For phosphoproteomics analysis in 3T3-L1 adipocytes treated with GSK3 inhibitor, labelfree quantification was applied and samples measured on a $\mathrm{Q}$ Exactive HF-X mass spectrometer (Thermo Fisher Scientific) [57] as previously described [58].

\section{Processing of spectral data}

Raw mass spectrometry data was processed using the Andromeda algorithm integrated into MaxQuant (v1.6.6.0 or v1.6.1.0) (Cox \& Mann, 2008), searching against the mouse UniProt database (June 2019 release) concatenated with known contaminants. Default settings were used, with the addition of Phospho(STY) in the variable modifications (in addition to the default, Oxidized Methionine (M) and Acetylation (Protein N-term)) for the phosphoproteomics study, or the addition of Phospho(STY), GlyGly(K), Oxidation(M), Acetyl(K), Deamidation(NQ), Methyl(KR) in the variable modifications for TRARG1 PTMomics study, and match between runs was turned on with a match time window of $0.7 \mathrm{~min}$ and an alignment time window of $20 \mathrm{~min}$ for identifications transfer between adjacent fractions, only for samples analyzed using the same nanospray conditions. For TRARG1 PTMomics, only mouse TRARG1 
bioRxiv preprint doi: https://doi.org/10.1101/2020.12.07.415257; this version posted December 7, 2020. The copyright holder for this preprint (which was not certified by peer review) is the author/funder, who has granted bioRxiv a license to display the preprint in perpetuity. It is made available under aCC-BY 4.0 International license.

sequence was used for searching. Protein, peptide and site FDR was filtered to $1 \%$ respectively.

\section{Quantification and statistical analysis}

\section{TRARG1 interactome}

This study was performed with three biological replicates. The values for each IgG replicate were generated by averaging each IgG replicate under the three conditions (basal, insulin and GSK3i) to eliminate variances between conditions driven by IgG control. Proteins were filtered out for reverse sequences, potential contaminants, peptides only identified by site and proteins with less than two quantified values in all bait conditions. LFQ intensities were $\log _{2}$-transformed and median normalized. Missing values were imputed as previously described [59]. Briefly, if values were quantified in at least two replicates for a condition, the remaining missing values would be imputed by sampling from a distribution with the same mean and standard deviation as the quantified replicates for that particular condition and protein. For remaining missing values in $\mathrm{IgG}$ controls, a second step of imputation was performed using a downshifting approach where missing values were replaced by sampling from a normal distribution that resembles the low tail of the distribution of quantified values in each sample (down shifted mean $=$ sample mean $-1 \mathrm{x}$ sample standard deviation, down shifted standard deviation $=0.6 \mathrm{x}$ sample standard deviation) $[60]$. To characterize the changes in protein abundance in bait condition compared to IgG control condition, $\log _{2}$ fold changes were estimated and two sample $t$ tests were performed for the proteins with no missing values after imputation. $p$ values were corrected for multiple hypothesis testing using the Benjamini and Hochberg method [61]. To determine which proteins are differently enriched in insulin or GSK3 inhibitor condition compared to basal condition, median absolute deviation (MAD) was calculated based on the variability of $\log _{2}$ fold change (Bait-IgG) of all quantified proteins between insulin/GSK3 inhibitor condition and basal condition. Proteins that have an absolute $\log _{2}$ fold change (Bait-IgG) difference greater than $2 * \mathrm{MAD}$ between the two compared conditions and $\log _{2}$ fold change (BaitIgG) greater than 2 and adj. $p \leq 0.05$ for either of the compared conditions are labelled as differently enriched in these two conditions (regulated by the treatment compared to basal condition).

\section{GSK3 inhibitor phosphoproteomic study}

This study was performed with four biological replicates. Phosphopeptides groups were filtered out for reverse sequences, potential contaminants and those only quantified 2 times or fewer out of the 8 samples. LFQ intensities were $\log _{2}-$ transformed and median normalized. Each group was imputed as previously described in [59]. For remaining missing values, a second step of imputation was performed if at least three replicates had quantified values in one condition and the values were completely missing or had only one quantified replicate in the other condition, using a method previously described in [60]. For the phosphopeptides with no missing values after imputation, two sample t tests were performed. Treatment was compared to control and $\mathrm{p}$ values were corrected for multiple hypothesis testing using the Benjamini and Hochberg method [61].

\section{Western blotting intensities and $q P C R$}

In phosphatase inhibitor assays, differences between control and treated intensities were tested with unpaired two-sided student's t-tests with Welch's correction. In kinase inhibitor assays, differences were tested by one-way ANOVA with Dunnett's multiple comparisons test. In tissue explants experiment, differences were tested with paired two-sided t-tests. In GSK3 knockdown experiments, differences were tested by RM one-way ANOVA with HolmSidak's multiple comparisons test. qPCR data were analyzed by two-way ANOVA with Dunnett's multiple comparisons test. Analyses were performed using GraphPad Prism version 8.0 for macOS (GraphPad Software, CA USA). Error bars are presented as standard error of the mean (SEM). Significance is represented, with a p-value $<0.05$ by $*,<0.01$ by $* *,<0.001$ by $* * *$ and $<0.0001$ by $* * * *$.

\section{Endogenous GLUT4 and HA-GLUT4-mRuby3 translocation assays}

For endogenous GLUT4 translocation assay, experimental values were normalized to the average value across all conditions in each experiment. Differences of surface GLUT4 relative to nuclear DNA among different conditions were tested using two-way ANOVA with correction for multiple comparisons. 
bioRxiv preprint doi: https://doi.org/10.1101/2020.12 07.415257 ; this version posted December 7,2020 . The copyright holder for this preprint (which was not certified by peer review) is the author/funder, who has granted bioRxiv a license to display the preprint in perpetuity. It is made available under aCC-BY 4.0 International license.

Differences of increase of surface GLUT4 relative to nuclear DNA among different conditions were tested using paired two-sided ttests. For HA-GLUT4-mRuby3 translocation assay, experimental values were normalized to the minimum and maximum values in control cells in each experiment. Differences of surface versus total GLUT4 between control and experimental condition were tested using twoway ANOVA with Dunnett's multiple comparisons test. Analyses were performed using GraphPad Prism version 8.0 for macOS (GraphPad Software, CA USA). Error bars are SEM. Significance is represented as stated in figure legends.

\section{${ }^{3} \mathrm{H}$-2-deoxyglucose uptake assay}

Differences of ${ }^{3} \mathrm{H}$-2-deoxyglucose uptake between control and experimental condition were tested by two-way ANOVA with Dunnett's multiple comparisons test. Analyses were performed using GraphPad Prism version 8.0 for macOS (GraphPad Software, CA USA). Error bars are SEM. Significance is represented as stated in figure legends.
Media glucose consumption/lactate production assay

Differences of Media glucose consumption/lactate production between control and experimental condition were tested by oneway ANOVA with Dunnett's multiple comparisons test. Analyses were performed using GraphPad Prism version 6.0 for Mac (GraphPad Software, CA USA). Error bars are SEM. Significance is represented, with a $\mathrm{p}<0.05$ by *, $<0.01$ by $* *,<0.001$ by $* * *$ and $<0.0001$ by $* * * *$.

Data availability: Raw and MaxQuant processed data of MS-based proteomics (except for the phosphoproteomic analysis of GSK3 inhibitor treated cells, which will be uploaded to accompany a future publication) have been deposited in the PRIDE ProteomeXchange Consortium

(http://proteomecentral.proteomexchange.org/cgi GetDataset) [62] and can be accessed with the identifier PXD022765. 
bioRxiv preprint doi: https://doi.org/10.1101/2020.12.07.415257; this version posted December 7, 2020. The copyright holder for this preprint (which was not certified by peer review) is the author/funder, who has granted bioRxiv a license to display the preprint in perpetuity. It is made available under aCC-BY 4.0 International license.

\section{Acknowledgements}

We thank Dr Joseph Rucker and Integral Molecular (PA, USA) for kindly providing the LM048 antiGLUT4 antibody and Professor Martin Wabitsch (Division of Pediatric Endocrinology and Diabetes, University Medical Center Ulm, Germany) for generously providing SGBS cells. These studies were supported by Sydney Mass Spectrometry, the Wellcome-MRC, Institute of Metabolic Science, Metabolic Research Laboratories, Imaging Core (Wellcome Trust Major Award [208363/Z/17/Z]) and the MRC MDU Mouse Biochemistry Laboratory [MRC_MC_UU_12012/5], Cambridge, for analysis of media glucose and lactate.

\section{Funding and additional information}

X.D. was supported by China Scholarship Council (CSC)-The University of Sydney (USYD) joint postgraduate scholarship (201606270221). W.P.B. and F.M.B. were supported by Wellcome Trust Investigator Award (107858/Z/15/Z) and UKRI-MRC grant MR/S008144/1 to F.M.B. B.L.P was supported by a National Health and Medical Research Council (NHMRC) Early Career Fellowship APP1072129. O.J.C was supported by a Wellcome Trust PhD studentship in Basic Science. J.G.B. was supported by a Diabetes Australia Research Program grant. J.R.K. was supported by a Diabetes Australia Research Program grant and an Australian Diabetes Society Skip Martin Early-Career Fellowship. D.E.J was supported by a NHMRC Senior Principal Research Fellowship APP1019680. D.J.F was supported by a Diabetes Australia Research Program grant and UKRI-MRC Career Development Award MR/S007091/1. The contents of the published material are solely the responsibility of the authors and do not reflect the views of the NHMRC.

Conflict of Interest: The authors declare no conflicts of interest in regards to this manuscript.

\section{References}

1. Stockli, J., D.J. Fazakerley, and D.E. James, GLUT4 exocytosis. J Cell Sci, 2011. 124(Pt 24): p. 4147-59.

2. Jedrychowski, M.P., et al., Proteomic analysis of GLUT4 storage vesicles reveals LRPI to be an important vesicle component and target of insulin signaling. J Biol Chem, 2010. 285(1): p. 104-14.

3. Hashiramoto, M. and D.E. James, Characterization of insulin-responsive GLUT4 storage vesicles isolated from 3T3-L1 adipocytes. Mol Cell Biol, 2000. 20(1): p. 416-27.

4. Larance, M., et al., Characterization of the role of the Rab GTPase-activating protein AS160 in insulin-regulated GLUT4 trafficking. J Biol Chem, 2005. 280(45): p. 37803-13.

5. $\quad$ Fazakerley, D.J., et al., Proteomic Analysis of GLUT4 Storage Vesicles Reveals Tumor Suppressor Candidate 5 (TUSC5) as a Novel Regulator of Insulin Action in Adipocytes. J Biol Chem, 2015. 290(39): p. 23528-42.

6. Beaton, N., et al., TUSC5 regulates insulin-mediated adipose tissue glucose uptake by modulation of GLUT4 recycling. Mol Metab, 2015. 4(11): p. 795-810.

7. $\quad \mathrm{Ng}, \mathrm{Y}$., et al., Rapid activation of Akt2 is sufficient to stimulate GLUT4 translocation in 3T3L1 adipocytes. Cell Metab, 2008. 7(4): p. 348-56.

8. Sano, H., et al., Insulin-stimulated phosphorylation of a Rab GTPase-activating protein regulates GLUT4 translocation. J Biol Chem, 2003. 278(17): p. 14599-602.

9. Sano, H., et al., Rab10, a target of the AS160 Rab GAP, is required for insulin-stimulated translocation of GLUT4 to the adipocyte plasma membrane. Cell Metab, 2007. 5(4): p. 293303.

10. Lansey, M.N., et al., Deletion of Rab GAP AS160 modifies glucose uptake and GLUT4 translocation in primary skeletal muscles and adipocytes and impairs glucose homeostasis. Am J Physiol Endocrinol Metab, 2012. 303(10): p. E1273-86.

11. Eguez, L., et al., Full intracellular retention of GLUT4 requires AS160 Rab GTPase activating protein. Cell Metab, 2005. 2(4): p. 263-72.

12. Kioumourtzoglou, D., G.W. Gould, and N.J. Bryant, Insulin stimulates syntaxin4 SNARE complex assembly via a novel regulatory mechanism. Mol Cell Biol, 2014. 34(7): p. 1271-9.

13. Cross, D.A., et al., Inhibition of glycogen synthase kinase-3 by insulin mediated by protein kinase B. Nature, 1995. 378(6559): p. 785-9. 
bioRxiv preprint doi: https://doi.org/10.1101/2020.12.07.415257; this version posted December 7, 2020. The copyright holder for this preprint (which was not certified by peer review) is the author/funder, who has granted bioRxiv a license to display the preprint in perpetuity. It is made available under aCC-BY 4.0 International license.

14. Beurel, E., S.F. Grieco, and R.S. Jope, Glycogen synthase kinase-3 (GSK3): regulation, actions, and diseases. Pharmacol Ther, 2015. 148: p. 114-31.

15. Lee, J. and M.S. Kim, The role of GSK3 in glucose homeostasis and the development of insulin resistance. Diabetes Res Clin Pract, 2007. 77 Suppl 1: p. S49-57.

16. Coghlan, M.P., et al., Selective small molecule inhibitors of glycogen synthase kinase-3 modulate glycogen metabolism and gene transcription. Chem Biol, 2000. 7(10): p. 793-803.

17. Cline, G.W., et al., Effects of a novel glycogen synthase kinase-3 inhibitor on insulinstimulated glucose metabolism in Zucker diabetic fatty (fa/fa) rats. Diabetes, 2002. 51(10): p. 2903-10.

18. Ring, D.B., et al., Selective glycogen synthase kinase 3 inhibitors potentiate insulin activation of glucose transport and utilization in vitro and in vivo. Diabetes, 2003. 52(3): p. 588-95.

19. Patel, S., et al., Tissue-specific role of glycogen synthase kinase 3beta in glucose homeostasis and insulin action. Mol Cell Biol, 2008. 28(20): p. 6314-28.

20. Summers, S.A., et al., The role of glycogen synthase kinase 3beta in insulin-stimulated glucose metabolism. J Biol Chem, 1999. 274(25): p. 17934-40.

21. Orena, S.J., A.J. Torchia, and R.S. Garofalo, Inhibition of glycogen-synthase kinase 3 stimulates glycogen synthase and glucose transport by distinct mechanisms in 3T3-L1 adipocytes. J Biol Chem, 2000. 275(21): p. 15765-72.

22. Dissanayake, W.C., et al., beta-catenin is important for the development of an insulin responsive pool of GLUT4 glucose transporters in 3T3-L1 adipocytes. Exp Cell Res, 2018. 366(1): p. 49-54.

23. Pearlman, S.M., Z. Serber, and J.E. Ferrell, Jr., A mechanism for the evolution of phosphorylation sites. Cell, 2011. 147(4): p. 934-46.

24. Humphrey, S.J., et al., Dynamic Adipocyte Phosphoproteome Reveals that Akt Directly Regulates mTORC2. Cell Metabolism, 2013. 17(6): p. 1009-1020.

25. Strumillo, M.J., et al., Conserved phosphorylation hotspots in eukaryotic protein domain families. Nat Commun, 2019. 10(1): p. 1977.

26. Duan, X., et al., Membrane Topology of Trafficking Regulator of GLUT4 1 (TRARG1). Biochemistry, 2018. 57(26): p. 3606-3615.

27. Nishi, H., K. Hashimoto, and A.R. Panchenko, Phosphorylation in protein-protein binding: effect on stability and function. Structure, 2011. 19(12): p. 1807-15.

28. Duan, Y., et al., Ubiquitin ligase RNF20/40 facilitates spindle assembly and promotes breast carcinogenesis through stabilizing motor protein Eg5. Nature Communications, 2016. 7.

29. Siegrist, F., M. Ebeling, and U. Certa, The small interferon-induced transmembrane genes and proteins. J Interferon Cytokine Res, 2011. 31(1): p. 183-97.

30. Weston, S., et al., A membrane topology model for human interferon inducible transmembrane protein 1. PLoS One, 2014. 9(8): p. e104341.

31. Even, A., et al., ATAT1-enriched vesicles promote microtubule acetylation via axonal transport. Sci Adv, 2019. 5(12): p. eaax2705.

32. Saadi, I., et al., Deficiency of the cytoskeletal protein SPECC1L leads to oblique facial clefting. Am J Hum Genet, 2011. 89(1): p. 44-55.

33. Sendamarai, A.K., et al., Structure of the membrane proximal oxidoreductase domain of human Steap3, the dominant ferrireductase of the erythroid transferrin cycle. Proc Natl Acad Sci U S A, 2008. 105(21): p. 7410-5.

34. Ohgami, R.S., et al., Identification of a ferrireductase required for efficient transferrindependent iron uptake in erythroid cells. Nat Genet, 2005. 37(11): p. 1264-9.

35. Gonzalez, E. and T.E. McGraw, Insulin signaling diverges into Akt-dependent and independent signals to regulate the recruitment/docking and the fusion of GLUT4 vesicles to the plasma membrane. Mol Biol Cell, 2006. 17(10): p. 4484-93.

36. Tokarz, V.L., P.E. MacDonald, and A. Klip, The cell biology of systemic insulin function. J Cell Biol, 2018. 217(7): p. 2273-2289.

37. Taelman, V.F., et al., Wnt signaling requires sequestration of glycogen synthase kinase 3 inside multivesicular endosomes. Cell, 2010. 143(7): p. 1136-48.

38. Humphrey, S.J., D.E. James, and M. Mann, Protein Phosphorylation: A Major Switch Mechanism for Metabolic Regulation. Trends Endocrinol Metab, 2015. 26(12): p. 676-687. 
bioRxiv preprint doi: https://doi.org/10.1101/2020.12.07.415257; this version posted December 7, 2020. The copyright holder for this preprint (which was not certified by peer review) is the author/funder, who has granted bioRxiv a license to display the preprint in perpetuity. It is made available under aCC-BY 4.0 International license.

39. Yaffe, M.B. and A.E. Elia, Phosphoserine/threonine-binding domains. Curr Opin Cell Biol, 2001. 13(2): p. 131-8.

40. Kramps, T., et al., Wnt/wingless signaling requires BCL9/legless-mediated recruitment of pygopus to the nuclear beta-catenin-TCF complex. Cell, 2002. 109(1): p. 47-60.

41. Xie, Y.Y., et al., Pygo2 Regulates Adiposity and Glucose Homeostasis via beta-CateninAxin2-GSK3beta Signaling Pathway. Diabetes, 2018. 67(12): p. 2569-2584.

42. $\quad$ Chaudhuri, R., et al., Cross-species gene expression analysis identifies a novel set of genes implicated in human insulin sensitivity. NPJ Syst Biol Appl, 2015. 1: p. 15010.

43. Masson, S.W.C., et al., beta-catenin regulates muscle glucose transport via actin remodelling and M-cadherin binding. Mol Metab, 2020: p. 101091.

44. Cantu, C., et al., A cytoplasmic role of Wnt/beta-catenin transcriptional cofactors Bcl9, Bcl9l, and Pygopus in tooth enamel formation. Sci Signal, 2017. 10(465).

45. Gibson, D.G., et al., Enzymatic assembly of DNA molecules up to several hundred kilobases. Nature Methods, 2009. 6(5): p. 343-U41.

46. Krycer, J.R., et al., The amino acid transporter, SLC1A3, is plasma membrane-localised in adipocytes and its activity is insensitive to insulin. Febs Letters, 2017. 591(2): p. 322-330.

47. Sanchis, J., et al., Improved PCR method for the creation of saturation mutagenesis libraries in directed evolution: application to difficult-to-amplify templates. Appl Microbiol Biotechnol, 2008. 81(2): p. 387-97.

48. Wabitsch, M., et al., Characterization of a human preadipocyte cell strain with high capacity for adipose differentiation. International Journal of Obesity, 2001. 25(1): p. 8-15.

49. Fischer-Posovszky, P., et al., Human SGBS Cells - a Unique Tool for Studies of Human Fat Cell Biology. Obesity Facts, 2008. 1(4): p. 184-189.

50. Katoh, K., J. Rozewicki, and K.D. Yamada, MAFFT online service: multiple sequence alignment, interactive sequence choice and visualization. Brief Bioinform, 2019. 20(4): p. 1160-1166.

51. Schindelin, J., et al., Fiji: an open-source platform for biological-image analysis. Nat Methods, 2012. 9(7): p. 676-82.

52. Tucker, D.F., et al., Isolation of state-dependent monoclonal antibodies against the 12transmembrane domain glucose transporter 4 using virus-like particles. Proceedings of the National Academy of Sciences of the United States of America, 2018. 115(22): p. E4990E4999.

53. Krycer, J.R., et al., Bicarbonate alters cellular responses in respiration assays. Biochem Biophys Res Commun, 2017. 489(4): p. 399-403.

54. Kunst, A., Drager, B, Ziegenhorn J., UV methods with hexokinase and glucose-6-phosphate dehrydrogenase. Methods of Enzymatic analysis, 1983. VI: p. 163-172.

55. Humphrey, S.J., et al., High-throughput and high-sensitivity phosphoproteomics with the EasyPhos platform. Nat Protoc, 2018. 13(9): p. 1897-1916.

56. Humphrey, S.J., S.B. Azimifar, and M. Mann, High-throughput phosphoproteomics reveals in vivo insulin signaling dynamics. Nat Biotechnol, 2015. 33(9): p. 990-5.

57. Kelstrup, C.D., et al., Performance Evaluation of the $Q$ Exactive HF-X for Shotgun Proteomics. J Proteome Res, 2018. 17(1): p. 727-738.

58. $\quad \mathrm{Su}, \mathrm{Z}$., et al., Global redox proteome and phosphoproteome analysis reveals redox switch in Akt. Nat Commun, 2019. 10(1): p. 5486.

59. Yang, P., et al., Multi-omic Profiling Reveals Dynamics of the Phased Progression of Pluripotency. Cell Syst, 2019. 8(5): p. 427-445 e10.

60. Robles, M.S., S.J. Humphrey, and M. Mann, Phosphorylation Is a Central Mechanism for Circadian Control of Metabolism and Physiology. Cell Metab, 2017. 25(1): p. 118-127.

61. Yoav Benjamini, Y.H., Controlling the False Discovery Rate: A Practical and Powerful Approach to Multiple Testing. Journal of the Royal Statistical Society: Series B (Methodological), 1995.

62. Perez-Riverol, Y., et al., The PRIDE database and related tools and resources in 2019: improving support for quantification data. Nucleic Acids Res, 2019. 47(D1): p. D442-D450.

63. Humphrey, S.J., et al., Dynamic adipocyte phosphoproteome reveals that Akt directly regulates $m$ TORC2. Cell Metab, 2013. 17(6): p. 1009-20. 
bioRxiv preprint doi: https://doi.org/10.1101/2020.12.07.415257; this version posted December 7, 2020. The copyright holder for this preprint (which was not certified by peer review) is the author/funder, who has granted bioRxiv a license to display the preprint in perpetuity. It is made available under aCC-BY 4.0 International license.

\section{Figures}

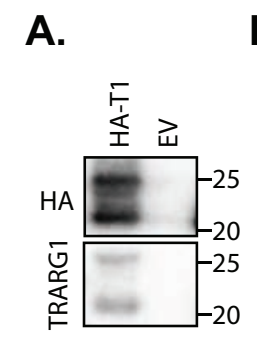

B.

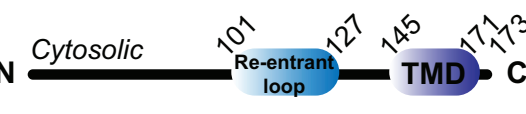

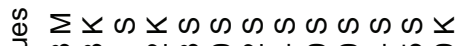

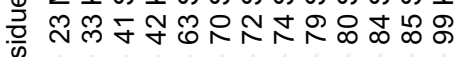

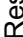

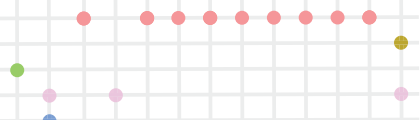

Phospho(STY)Sites • Oxidation(M)Sites

- GlyGly(K)Sites

- Acetyl(K)Sites
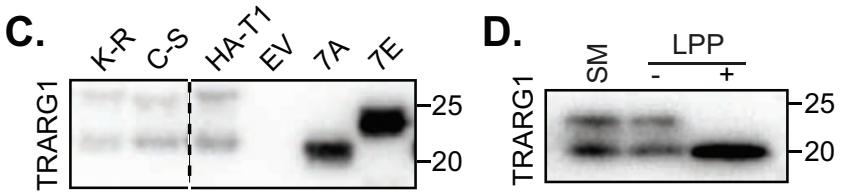

E.

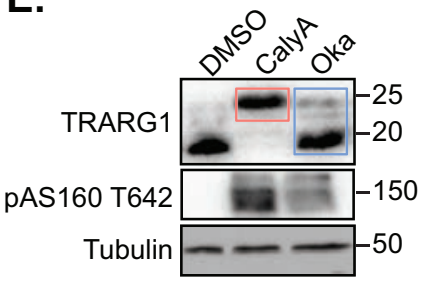

$\mathbf{F}$.

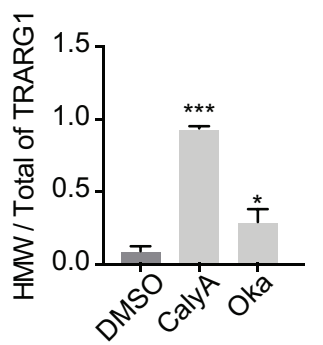

Figure 1. TRARG1 phosphorylation causes apparent higher molecular weight bands by immunoblotting. (A) HA-tagged TRARG1 (HA-TRARG1) expressed in HEK-293E cells shows multiple bands by immunoblotting. (B) PTMs detected by mass spectrometry on HA-TRARG1 (mouse) overexpressed in HEK-293E cells. (C) HA-TRARG1 phosphomutants with Ser/Thr mutated to Ala or Glu (7A/7E) expressed in HEK-293E cells exhibited a molecular weight similar to the apparent lower or higher molecular weight of HA-TRARG1, respectively. (D) In vitro Lambda protein phosphatase treatment of 3T3-L1 adipocytes lysates removed apparent higher molecular weight TRARG1 bands. (E) Apparent higher molecular weight TRARG1 bands were increased in intensity in 3T3-L1 adipocytes treated with phosphatase inhibitors, calyculin A or okadaic acid. (F) Quantification of (E). The ratio of apparent higher molecular weight TRARG1 (as indicated by the bands in the pink box in (E)) signal to total TRARG1 (as indicated by the bands in the blue box in (E)) signal was quantified as a metric of TRARG1 phosphorylation ( $\mathrm{n}=3$, mean $\pm \mathrm{SEM},{ }^{*} p<0.05$; ${ }^{* * *} p<0.001$, comparisons with cells under DMSO condition). 
bioRxiv preprint doi: https://doi.org/10.1101/2020.12.07.415257; this version posted December 7, 2020. The copyright holder for this preprint (which was not certified by peer review) is the author/funder, who has granted bioRxiv a license to display the preprint in perpetuity. It is made available under aCC-BY 4.0 International license.

A.

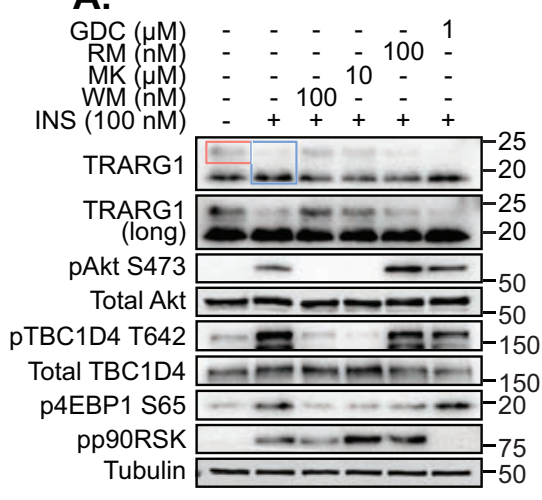

B.

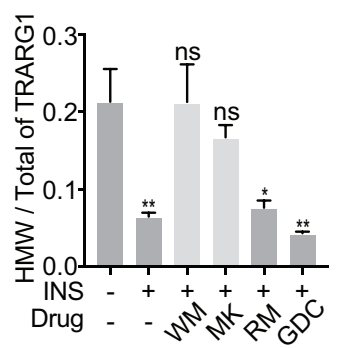

c.

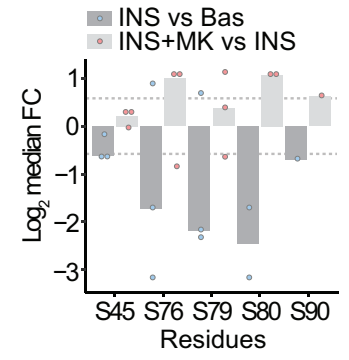

D.

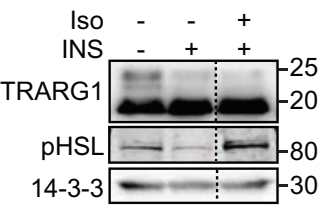

F.

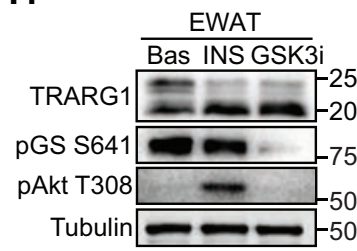

I.

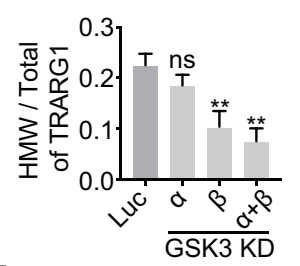

K.

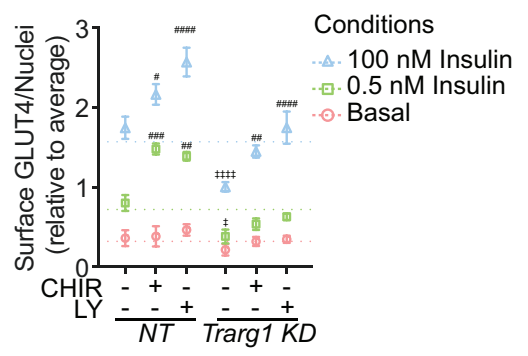

E.

G.

J.
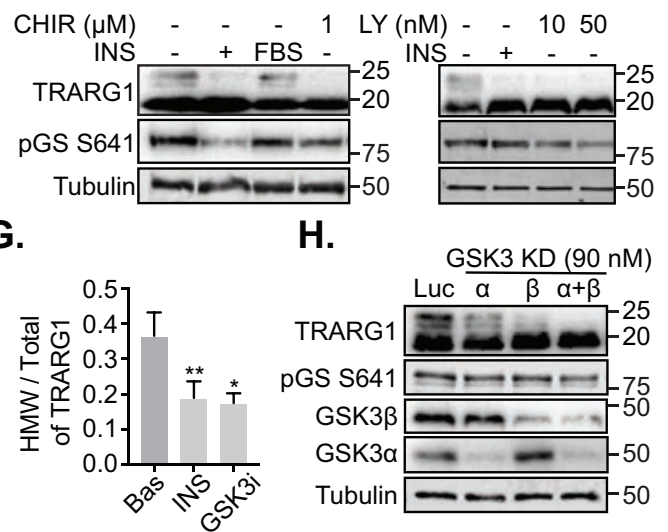

H. GSK3 KD $(90 \mathrm{nM})$ $\begin{aligned} & \text { Luc } \alpha \quad \beta \alpha+\beta \\ \text { TRARG1 } & \\ \text { PGS S641 } & \\ \text { GSK3 } 30 & \\ \text { GSK3a } & \\ \text { Tubulin } & \end{aligned}$

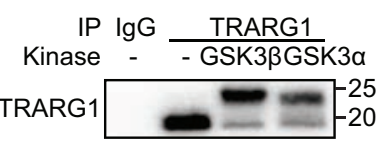

L.

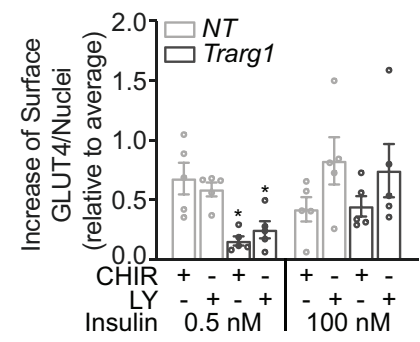

Figure 2. TRARG1 is dephosphorylated with insulin through PI3K/Akt/GSK3 axis. (A) 3T3-L1 adipocytes were serum-starved prior to insulin stimulation $(100 \mathrm{nM}, 20 \mathrm{~min})$. Where indicated, a PI3K inhibitor (wortmannin, $100 \mathrm{nM}$ ), Akt inhibitor (MK-2206, $10 \mu \mathrm{M}$ ), mTOR inhibitor (rapamycin, 100 $\mathrm{nM}$ ), or MAPK inhibitor (GDC-0994, $1 \mu \mathrm{M}$ ) was administered 20 min prior to insulin treatment. Samples were analyzed by immunoblotting. (B) Quantification of (A). The ratio of apparent higher molecular weight TRARG1 (as indicated by the bands in the pink box in (A)) signal to total TRARG1 (as indicated by the bands in the blue box in (A)) signal was quantified as a metric of TRARG1 phosphorylation ( $\mathrm{n}=3$, mean $\pm \mathrm{SEM},{ }^{*} p<0.05$; $* *<<0.01$; ns, non-significant, comparisons with cells without insulin or drug treatment). (C) Bar plot indicating the $\log _{2}$-transformed median FC in phosphorylation of insulin versus basal or insulin+MK versus insulin alone at Class I TRARG1 phosphosites reported by Humphrey et al. [63]. SILAC-labelled adipocytes were serum-starved prior to insulin stimulation (100 nM, $20 \mathrm{~min})$. The Akt inhibitor, MK-2206 $(10 \mu \mathrm{M})$, was administered 30 min prior to insulin treatment where indicated. Only those sites downregulated $\leq 0.67$-fold in median following insulin stimulation are shown. Dashed lines indicate where $\log _{2} \mathrm{FC}=0.58$ or -0.58 . (D) 3T3L1 adipocytes were serum-starved, followed by treatment with insulin (INS; $100 \mathrm{nM})$ or insulin (100 $\mathrm{nM}$ ) and isoproterenol (Iso; $1 \mathrm{nM}$ ) for $20 \mathrm{~min}$. Samples were analyzed by immunoblotting. (E) 3T3-L1 adipocytes were serum-starved (except for the $F B S$ lane), followed by treatment with insulin $(100 \mathrm{nM})$, CHIR99021 (CHIR) (left panel) or LY2090314 (LY) (right panel) at indicated doses for $20 \mathrm{~min}$. Samples were analyzed by immunoblotting. (F) Epididymal white adipose tissue (EWAT) was excised from mice and minced. Explants were serum-starved in DMEM/2\% BSA/20 mM HEPES, pH 7.4 for 2 $\mathrm{h}$ followed by treatment with insulin $(10 \mathrm{nM})$ or LY2090314 (GSK3i) $(500 \mathrm{nM})$ for 30 min at $37^{\circ} \mathrm{C}$. Treatment was terminated and tissues were solubilized in RIPA buffer and subjected to analysis by immunoblotting. (G) Quantification of (F). The ratio of apparent higher molecular weight TRARG1 signal to total TRARG1 signal was quantified $\left(\mathrm{n}=4\right.$, mean $\pm \mathrm{SEM},{ }^{*} p<0.05 ; * * p<0.01 ; * * * p<0.001$, comparisons with basal condition). (H) 3T3-L1 adipocytes on day 6 post-differentiation were 
bioRxiv preprint doi: https://doi org/10.1101/2020 12 07.415257: this version posted December 7, 2020. The copyright holder for this preprint (which was not certified by peer review) is the author/funder, who has granted bioRxiv a license to display the preprint in perpetuity. It is made available under aCC-BY 4.0 International license.

transfected with esiRNA targeting GSK3 $\alpha$ and/or $\beta$. Cells transfected with esiRNA targeting luciferase (Luc) were used as control. $72 \mathrm{~h}$ post-transfection, cells were serum-starved for $2 \mathrm{~h}$ and harvested for analysis by immunoblotting. (I) Quantification of $(\mathrm{H})$. The level of TRARG1 phosphorylation, glycogen synthase phosphorylation, GSK3 $\alpha$ and GSK3 $\beta$ in $(\mathrm{H})$ were quantified $(\mathrm{n}=3$, mean $\pm \mathrm{SEM}, * p$ $<0.05 ; * *<<0.01$, comparisons with cells transfected with esiRNA targeting luciferase). (J) 3T3-L1 adipocytes were serum-starved in basal DMEM media containing $100 \mathrm{nM}$ LY2090314 for $2 \mathrm{~h}$. Cells were lysed and homogenized and cell lysates were immunoprecipitated with anti-TRARG1 antibody or IgG as a control. Immunoprecipitated TRARG1 was treated with GSK $3 \alpha, \beta$ or reaction buffer alone. All samples were analyzed by immunoblotting. (K) 3T3-L1 adipocytes were serum-starved in the absence (DMSO) or presence of GSK3 inhibitors (10 $\mu$ M CHIR99021; CHIR or 500 nM LY2090314; LY) before treated with or without $0.5 \mathrm{nM}$ or $100 \mathrm{nM}$ insulin for $20 \mathrm{~min}$. Surface GLUT4 detected by immuno-labelling relative to nuclear DNA level is shown ( $\mathrm{n}=5$, mean $\pm \mathrm{SEM},{ }^{\#} p<0.05,{ }^{\# \#} p<0.01,{ }^{\# \#} p$ $<0.001, \ldots+0.0001$, compared to the DMSO condition with the same insulin treatment and gene knockdown; ${ }^{\star} p<0.05,{ }^{4+1} p<0.0001$, compared to the non-targeting (NT) knockdown with the same insulin and drug treatment). (L) Differences in PM GLUT4 (relative to nuclear DNA) between CHIRor LY-treated and DMSO control condition with the same insulin treatment and gene knockdown as shown in (K) were calculated (mean $\pm \mathrm{SEM},{ }^{*} p<0.05$, compared to NT knockdown with the same insulin and drug treatment). 
bioRxiv preprint doi: https://doi.org/10.1101/2020.12.07.415257; this version posted December 7, 2020. The copyright holder for this preprint (which was not certified by peer review) is the author/funder, who has granted bioRxiv a license to display the preprint in perpetuity. It is made available under aCC-BY 4.0 International license.

A.

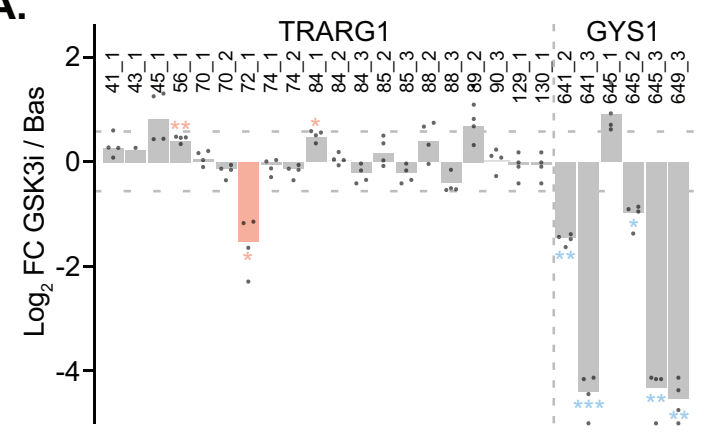

E.

TRARG1

$\mathbf{N}$

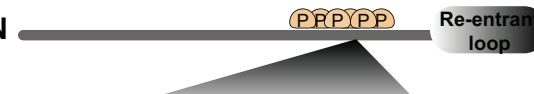

72_APLPRSPSRASSRRASSIATTSY_94 Homo sapiens

69 -PSLSGSPSRVSSRRASSVITTSY_91 Mus musculus

69_PSLSGSPSRASSRRASSVVTTSY_91 Rattus norvegicus

72_ALHPLSSSRASSRRASSTATTSY 94 Bos taurus

74 A-APLSPSRLSLGRASSTATSTA 95 Gallus gallus

79_S-APLSPSRVSLARASSTATT--_98 Xenopus tropicalis

74 A-APLSPSRVSLSRTSSTGNA-- 93 Danio rerio(a)

64 _ASVPRSSSRASFRCASPP-----_81 Danio rerio(b)

81_A-PPGSPSRVSLGRVSTTTNS--_100Callorhinchus milii

F.

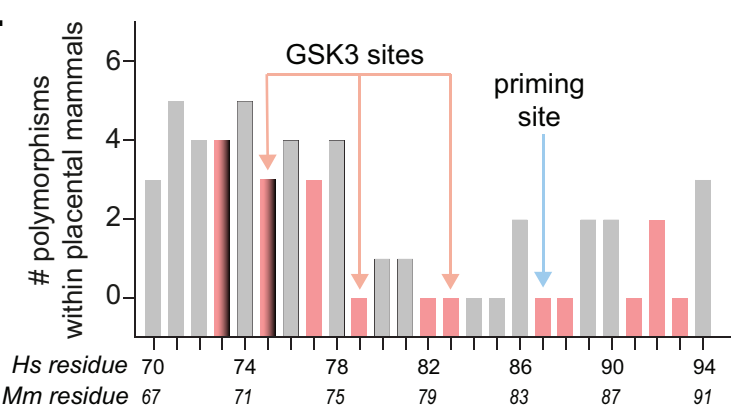

B.
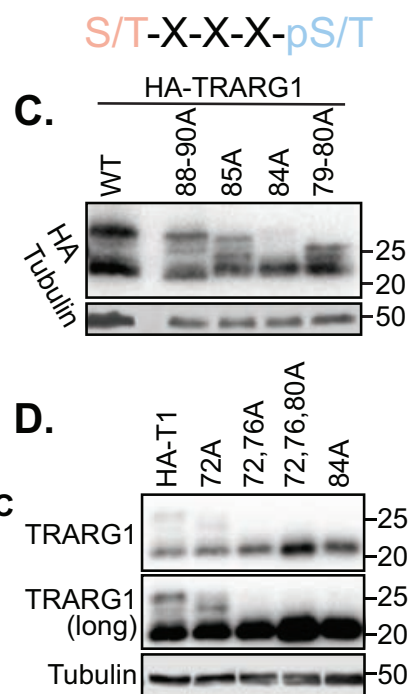

G.

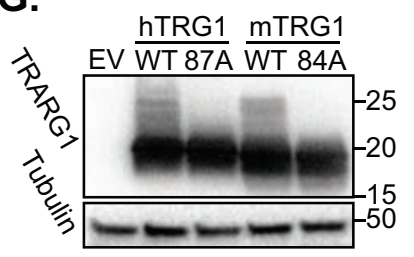

Figure 3. Mouse TRARG1 is primed at $\mathbf{S 8 4}$ for subsequent phosphorylation by GSK3 within a highly conserved region. (A) 3T3-L1 adipocytes were serum-starved followed by treatment with GSK3 inhibitor LY2090314 (100 nM, $20 \mathrm{~min})$ or DMSO as a control. Cells were subjected to phosphoproteomic analysis. Bar plot of $\log _{2}$-transformed mean FC of all detected TRARG1 sites and S641, S645 and S649 on glycogen synthase from this analysis is shown. Numbers following underscore indicate the number of phosphorylation sites detected on that peptide (significance is indicated by *adj. $p<0.05$; **adj. $p<0.01$; ***adj. $p<0.001$, t test). (B) GSK3 substrate consensus motif. Prephosphorylated (primed) site is labeled in blue and GSK3 target site is labeled in pink. (C) Mouse HATRARG1 phosphomutants generated by mutating T88, T89 and S90 to Ala (88-90A), S85 to Ala (85A), S84 to Ala (84A) or S79 and S80 to Ala (79-80A), or wild type HA-TRARG1 were transfected into HEK-293E cells. Cells were lysed $24 \mathrm{~h}$ post-transfection and samples were analyzed by immunoblotting. (D) Mouse HA-TRARG1 phosphomutants generated by mutating S72 to Ala (72A), S72 and S76 to Ala (72,76A), S72, S76 and S80 to Ala (72,76,80A) or S84 to Ala (84A), or wild type HA-TRARG1 were transfected in HEK-293E cells. Cells were lysed $24 \mathrm{~h}$ post-transfection and samples were analyzed by immunoblotting. (E) The phosphosite-rich region between residue 69 and 91 on mouse TRARG1 is highly conserved across species. Insulin/GSK3 regulated sites are labeled in red. Other conserved $\mathrm{Ser} / \mathrm{Thr}$ residues within this region are labeled in pink. (F) Polymorphism analysis of TRARG1 residues within 64 placental mammals. Ser/Thr residues conserved across mouse and human TRARG1 are colored in pink; Bars colored in pink with shade indicate Ser/Thr residues present in mouse but not human TRARG1 sequence. A full list of species included in the analysis is provided in Supplemental table S2. $H s$, Homo sapiens; $M m$, Mus musculus. (G) Wild type mouse TRARG1, mouse TRARG1 
bioRxiv preprint doi: https://doi.org/10.1101/2020.12.07.415257; this version posted December 7, 2020. The copyright holder for this preprint (which was not certified by peer review) is the author/funder, who has granted bioRxiv a license to display the preprint in perpetuity. It is made available under aCC-BY 4.0 International license.

with S84 mutated to Ala (84A), wild type human TRARG1, human TRARG1 with S87 (equivalent to S84 in mouse TRARG1) mutated to Ala (87A) were expressed in HEK-293E cells. Cells were lysed 24 $\mathrm{h}$ post-transfection and samples were analyzed by immunoblotting. 
bioRxiv preprint doi: https://doi.org/10.1101/2020.12.07.415257; this version posted December 7, 2020. The copyright holder for this preprint (which was not certified by peer review) is the author/funder, who has granted bioRxiv a license to display the preprint in perpetuity. It is made available under aCC-BY 4.0 International license.

A.

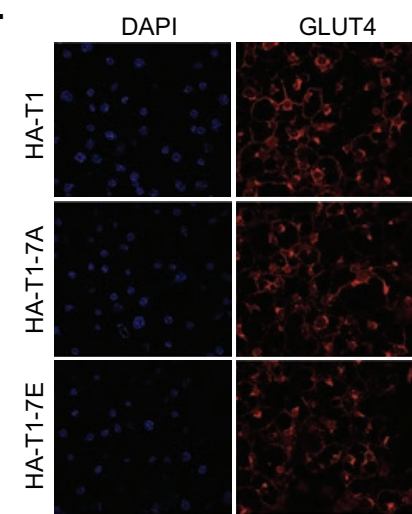

C.

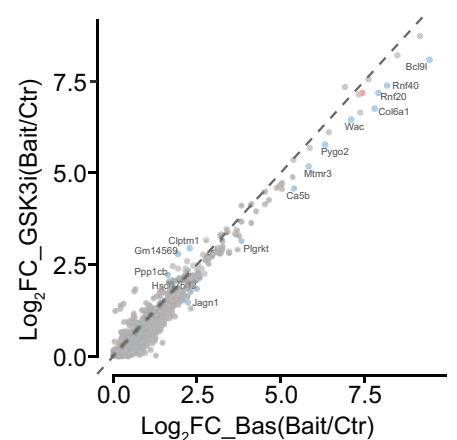

HA

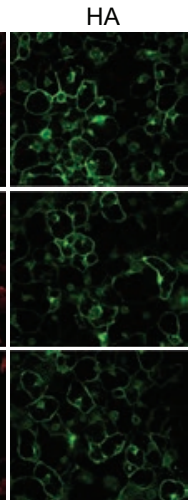

D.
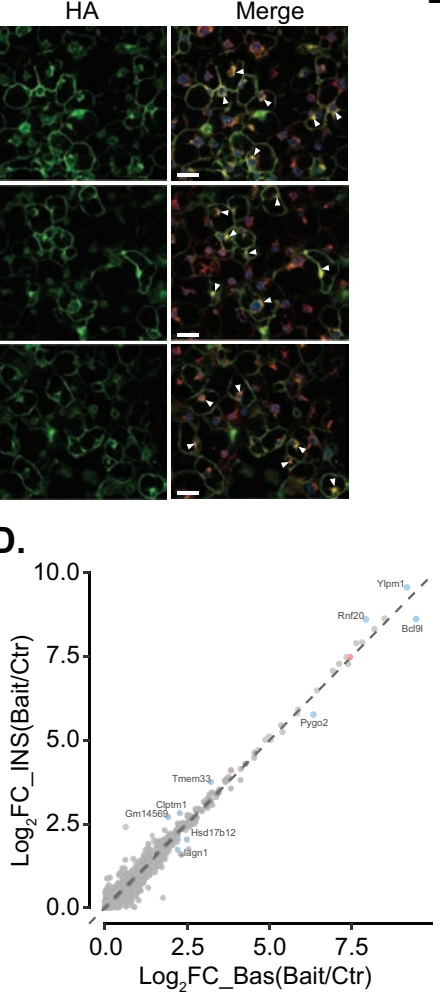

B.

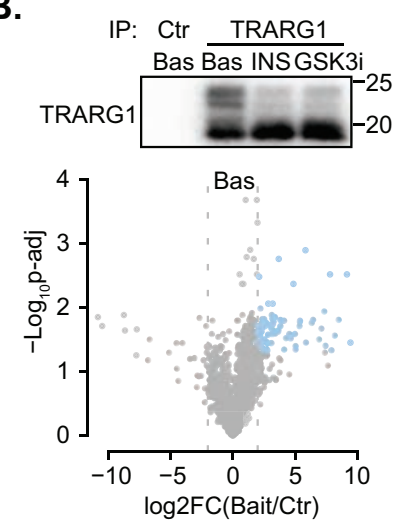

E.

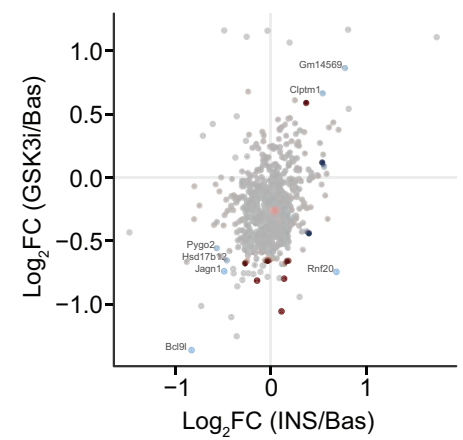

Figure 4. TRARG1 phosphorylation status regulates its interacting proteins but not its subcellular localization. (A) 3T3-L1 adipocytes stably expressing HA-TRARG1, HA-TRARG1-7A or HA-TRARG1-7E were serum-starved for $2 \mathrm{~h}$. Cells were fixed and stained for nuclei (DAPI, blue), GLUT4 (red) and HA (green). Immunofluorescence imaging was performed by confocal microscopy. Instances of colocalization between GLUT4 and HA-TRARG1, HA-TRARG1-7A or HA-TRARG1$7 \mathrm{E}$ are indicated by closed arrowheads. Scale bar, $20 \mu \mathrm{m}$. (B) Volcano plot of TRARG1 interactome under basal (Bas) condition. (C-D) TRARG1 interactors regulated by GSK3 inhibitor treatment (highlighted in blue in (C)) or insulin treatment (highlighted in blue in (D)) as identified by MAD analysis (4.22.2.), TRARG1 labeled in pink. (E) TRARG1 interactors regulated only by GSK3 inhibitor or insulin or by both treatments labeled in dark red, dark blue, or blue, respectively. TRARG1 labeled in pink. 
bioRxiv preprint doi: https://doi.org/10.1101/2020.12.07.415257; this version posted December 7, 2020. The copyright holder for this preprint (which was not certified by peer review) is the author/funder, who has granted bioRxiv a license to display the preprint in perpetuity. It is made available under aCC-BY 4.0 International license.

A.

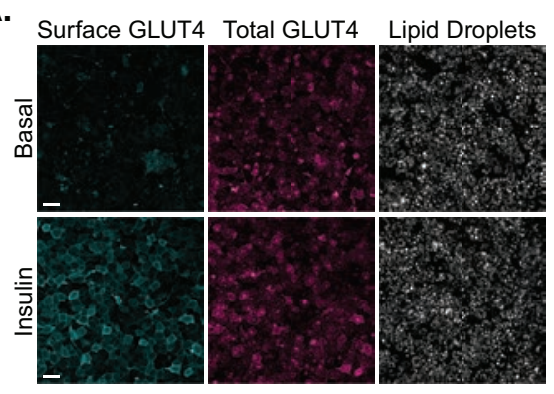

C.

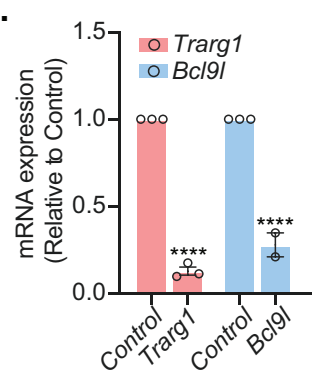

D.

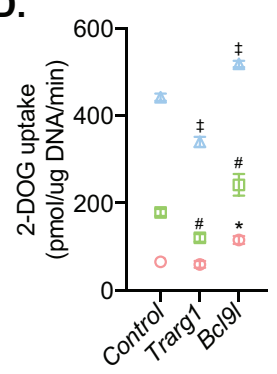

B.

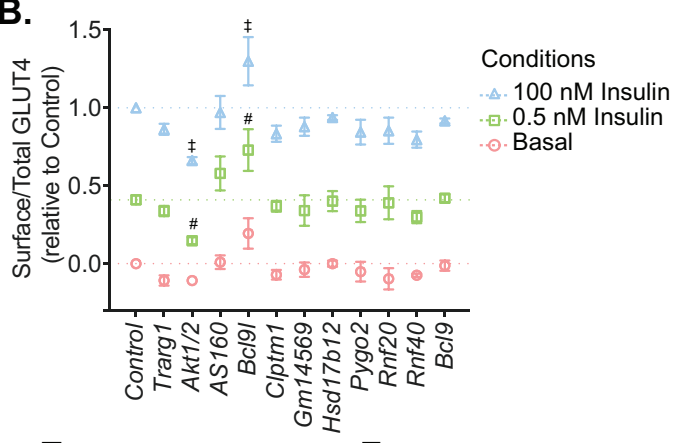

E.

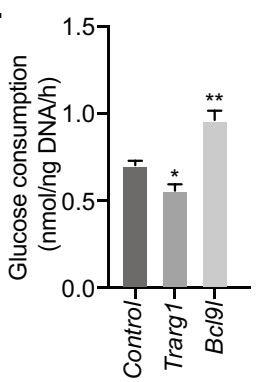

F.

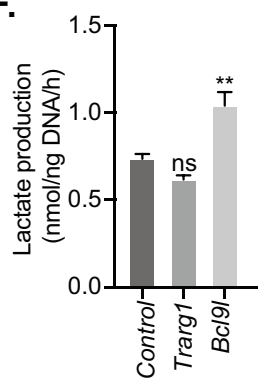

Figure 5. GLUT4 reporter translocation assay with the knockdown of selected TRARG1 interactors. (A) Representative images of HA-GLUT4-mRuby3 assay in 3T3-L1 adipocytes. Surface GLUT4 is indicated by fluorescently-labelled HA signal. Total GLUT4 is indicated by the mRuby3 signal. Digital phase contrast images highlight the lipid droplets present in the cells. Scale bar, $50 \mu \mathrm{m}$. (B) GLUT4 trafficking screen with the knockdown of selected TRARG1 interactors and positive controls (comparisons are with control in the same treatment condition, significance is indicated by $* p$ $<0.05$ for basal condition; ${ }^{\#} p<0.05$ for $0.5 \mathrm{nM}$ insulin condition; ${ }^{\sharp} p<0.05$ for $100 \mathrm{nM}$ insulin condition). (C) Knockdown efficiency of Trargl and Bcl9l as assessed by qPCR (**** $<0.0001$, comparison with control under the same knockdown condition). (D) 2-DOG uptake in 3T3-L1 adipocytes with the knockdown of selected TRARG1 interactors. The same legend is used as in Fig. 5B. (E) Media glucose consumption in 3T3-L1 adipocytes with the knockdown of Trargl and Bcl9l. (F) Media lactate production in 3T3-L1 adipocytes with the knockdown of Trarg1 and Bcl9l. 